\title{
ESTIMATES FOR THE LARGE TIME BEHAVIOR OF THE LANDAU EQUATION IN THE COULOMB CASE
}

\author{
K. CARrapatoso, L. Desvillettes, AND L. HE
}

\begin{abstract}
This work deals with the large time behaviour of the spatially homogeneous Landau equation with Coulomb potential. Firstly, we obtain a bound from below of the entropy dissipation $D(f)$ by a weighted relative Fisher information of $f$ with respect to the associated Maxwellian distribution, which leads to a variant of Cercignani's conjecture thanks to a logarithmic Sobolev inequality. Secondly, we prove the propagation of polynomial and stretched exponential moments with an at most linearly growing in time rate. As an application of these estimates, we show the convergence of any ( $H$ - or weak) solution to the Landau equation with Coulomb potential to the associated Maxwellian equilibrium with an explicitly computable rate, assuming initial data with finite mass, energy, entropy and some higher $L^{1}$-moment. More precisely, if the initial data have some (large enough) polynomial $L^{1}$-moment, then we obtain an algebraic decay. If the initial data have a stretched exponential $L^{1}$-moment, then we recover a stretched exponential decay.
\end{abstract}

\section{INTRODUCTION}

The Landau equation is a fundamental model in kinetic theory that describes the evolution in time of a plasma due to collisions between charged particles.

We consider in this work the spatially homogeneous Landau equation with Coulomb potential (cf. [28, 12, 20])

$$
\partial_{t} f=Q(f, f)
$$

which is complemented with initial data $f_{0}=f_{0}(v) \geq 0$. Here $f:=f(t, v) \geq 0$ stands for the distribution of particles that at time $t \in \mathbb{R}_{+}$possess the velocity $v \in \mathbb{R}^{3}$. The Landau operator $Q$ is a bilinear operator acting only on the velocity variable $v$. It writes

$$
Q(f, f)(v)=\nabla \cdot \int_{\mathbb{R}^{3}} a(v-w)\{f(w) \nabla f(v)-\nabla f(w) f(v)\} d w,
$$

where $a$ is a matrix-valued function that is symmetric, (semi-definite) positive. It depends on the interaction potential between particles, and is defined by (for $i, j=1,2,3$ )

$$
a_{i j}(z)=|z|^{\gamma+2} \Pi_{i j}(z), \quad \Pi_{i j}(z)=\delta_{i j}-\frac{z_{i} z_{j}}{|z|^{2}}, \quad-4<\gamma \leq 1
$$

Observe that $\Pi(z):=\left(\Pi_{i j}(z)\right)_{i, j=1,2,3}$ is the orthogonal projection onto $z^{\perp}$. One usually classifies the different cases as follows: hard potentials $0<\gamma \leq 1$, Maxwellian molecules $\gamma=0$, moderately soft potentials $-2 \leq \gamma<0$ and very soft potentials $-4<\gamma<-2$. Note that the very soft potentials include the Coulomb potential $\gamma=-3$. From now on, for the sake of clarity and because it is the most physically interesting case, we shall only consider in this work the Coulomb potential case $\gamma=-3$, except when moment estimates are concerned. It is however

Date: October 27, 2018.

2010 Mathematics Subject Classification. 35B65, 35K67, 45G05, 76P05, 82C40, 82D10.

Key words and phrases. Landau equation, Landau operator, entropy dissipation, degenerate diffusion, Coulomb interaction, large time behaviour, convergence to equilibrium. 
worth mentioning that our methods and results can be straightforwardly adapted to the soft potentials case $-4<\gamma<0$, as explained in more details in the remarks after our main theorems.

One usually introduces the quantities

$$
b_{i}(z)=\sum_{j=1}^{3} \partial_{j} a_{i j}(z)=-2 z_{i}|z|^{-3}, \quad c(z)=\sum_{i=1}^{3} \sum_{j=1}^{3} \partial_{i j} a_{i j}(z)=-8 \pi \delta_{0}(z),
$$

so that the Landau operator can be written as

$$
\begin{aligned}
Q(f, f) & =\sum_{i=1}^{3} \partial_{i}\left(\sum_{j=1}^{3}\left(a_{i j} * f\right) \partial_{j} f-\left(b_{i} * f\right) f\right) \\
& =\sum_{i=1}^{3} \sum_{j=1}^{3}\left(a_{i j} * f\right) \partial_{i j} f+8 \pi f^{2} .
\end{aligned}
$$

At the formal level, we can write thanks to (2) a weak formulation of the Landau operator $Q$, for a test function $\varphi$, in the following way:

$$
\begin{aligned}
\int_{\mathbb{R}^{3}} Q(f, f)(v) \varphi(v) d v \\
\quad=-\frac{1}{2} \sum_{i=1}^{3} \sum_{j=1}^{3} \iint_{\mathbb{R}^{3} \times \mathbb{R}^{3}} a_{i j}(v-w)\left\{\frac{\partial_{i} f}{f}(v)-\frac{\partial_{i} f}{f}(w)\right\}\left\{\partial_{j} \varphi(v)-\partial_{j} \varphi(w)\right\} f(v) f(w) d v d w .
\end{aligned}
$$

Another weak formulation, based on (44), also holds at the formal level:

$$
\begin{aligned}
\int_{\mathbb{R}^{3}} Q(f, f)(v) \varphi(v) d v= & \frac{1}{2} \sum_{i=1}^{3} \sum_{j=1}^{3} \iint_{\mathbb{R}^{3} \times \mathbb{R}^{3}} a_{i j}(v-w)\left\{\partial_{i j} \varphi(v)+\partial_{i j} \varphi(w)\right\} f(v) f(w) d v d w \\
& +\sum_{i=1}^{3} \iint_{\mathbb{R}^{3} \times \mathbb{R}^{3}} b_{i}(v-w)\left\{\partial_{i} \varphi(v)-\partial_{i} \varphi(w)\right\} f(v) f(w) d v d w .
\end{aligned}
$$

From the weak formulation (5), we can easily deduce some fundamental properties of the Landau operator $Q$. The operator indeed conserves (at the formal level) mass, momentum and energy, more precisely

$$
\int_{\mathbb{R}^{3}} Q(f, f)(v) \varphi(v) d v=0 \quad \text { for } \quad \varphi(v)=1, v_{i}, \frac{|v|^{2}}{2} .
$$

We also deduce from (5), at the formal level, the entropy structure by taking the test function $\varphi(v)=\log f(v)$, that is

$$
\begin{gathered}
D(f):=-\int Q(f, f)(v) \log f(v) d v \\
=\frac{1}{2} \sum_{i=1}^{3} \sum_{j=1}^{3} \iint_{\mathbb{R}^{3} \times \mathbb{R}^{3}} a_{i j}(v-w)\left\{\frac{\partial_{i} f}{f}(v)-\frac{\partial_{i} f}{f}(w)\right\}\left\{\frac{\partial_{j} f}{f}(v)-\frac{\partial_{j} f}{f}(w)\right\} f(v) f(w) d v d w .
\end{gathered}
$$

Note that $D(f) \geq 0$ since the matrix $a$ is (semi-definite) positive. It also follows (see for example 21]) that any equilibrium (that is, any $f$ such that $D(f)=0$ ) is a Maxwellian distribution

$$
\mu_{\rho, u, T}(v)=\frac{\rho}{(2 \pi T)^{3 / 2}} e^{-\frac{|v-u|^{2}}{2 T}},
$$


where $\rho \geq 0$ is the density, $u \in \mathbb{R}^{3}$ the mean velocity and $T>0$ the temperature, defined by

$$
\rho=\int_{\mathbb{R}^{3}} f(v) d v, \quad u=\frac{1}{\rho} \int_{\mathbb{R}^{3}} v f(v) d v, \quad T=\frac{1}{3 \rho} \int_{\mathbb{R}^{3}}|v-u|^{2} f(v) d v .
$$

As a consequence of the properties above at the level of the operator, we can obtain the corresponding properties (at the formal level) for the solutions of the spatially homogeneous Landau equation (1), that is, the conservation of mass, momentum and energy

$$
\forall t \geq 0, \quad \rho(t)=\rho(0), \quad u(t)=u(0), \quad T(t)=T(0),
$$

on one hand, and the entropy property on the other hand

$$
\frac{d}{d t} H(f(t, \cdot))=-D(f(t, \cdot)) \leq 0,
$$

where $H(f):=\int f(v) \log f(v) d v$ is the entropy and $D(f)$, defined by (8), is the entropy dissipation.

Throughout this paper, we shall always assume that $f_{0} \geq 0$ and $f_{0} \in L_{2}^{1} \cap L \log L\left(\mathbb{R}^{3}\right)$. Furthermore, in most of the paper, we suppose, without loss of generality, that $f_{0}$ satisfies the normalization identities

$$
\int_{\mathbb{R}^{3}} f_{0}(v) d v=1, \quad \int_{\mathbb{R}^{3}} f_{0}(v) v d v=0, \quad \int_{\mathbb{R}^{3}} f_{0}(v)|v|^{2} d v=3,
$$

which can be rewritten $\rho(0)=1, u(0)=0, T(0)=1$. Finally, we denote by $\mu(v)=(2 \pi)^{-3 / 2} e^{-|v|^{2} / 2}$ the Maxwellian distribution (centred reduced Gaussian) with same mass, momentum and energy as $f_{0}$ satisfying (12).

Let us briefly recall some existing results on the Landau equation (1) with Coulomb potential. Villani 36 proved global existence of the so-called $H$-solutions for initial data with finite mass, energy and entropy. More recently, the second author 14 proved that $H$-solutions are in fact weak solutions (in the usual sense), thanks to a new estimate for the entropy dissipation $D(f)$. More precisely it is obtained in 14 that there is an explicitly computable constant $C_{0}=C_{0}(\bar{H})>0$ such that, for all (normalized) $f \geq 0$ satisfying $H(f) \leq \bar{H}$, the following inequality holds:

$$
\|f\|_{L_{-3}^{3}} \leq C_{0}(1+D(f))
$$

where (for any $p \in\left[1,+\infty\left[, q \in \mathbb{R}\right.\right.$ ) the $L_{q}^{p}$ norm is defined by

$$
\|f\|_{L_{q}^{p}}^{p}=\int_{\mathbb{R}^{3}}|f(v)|^{p}\left(1+|v|^{2}\right)^{p q / 2} d v .
$$

Therefore, since (for $H$ solutions of the spatially homogeneous Landau equation) $D(f) \in$ $L_{t}^{1}(] 0, \infty[)$ thanks to identity (11), we obtain that any $H$ solution of this equation lies in $L_{l o c}^{1}\left([0, \infty) ; L_{-3}^{3}\left(\mathbb{R}^{3}\right)\right.$ ), which is sufficient to define weak solutions in the usual sense (using the weak form (6)), see [14 for more details. We also quote [37 for renormalized solutions in the spatially inhomogeneous context, and 2] for local in time solutions.

Let us mention the results concerning the well-posedness issue. Fournier 23 ] obtained that uniqueness holds in the class $L_{l o c}^{\infty}\left([0, \infty) ; L_{2}^{1}\left(\mathbb{R}^{3}\right)\right) \cap L_{l o c}^{1}\left([0, \infty) ; L^{\infty}\left(\mathbb{R}^{3}\right)\right)$, and this result implies a local well-posedness result assuming further that the initial data lie in $L^{\infty}\left(\mathbb{R}^{3}\right)$, thanks to the local existence result of Arsenev-Peskov [6] for such initial data. We also refer to [25] and [26] for the global well-posedness and the local well-posedness for the inhomogeneous equation in weighted Sobolev spaces, as well as to [3, 4, 24, for the non-cutoff Boltzmann equation, whose structure shares similarities with the Landau equation.

Concerning the large time behaviour issue, we shall mention some known results for all kind of potentials. In the spatially homogeneous case, Villani and the second author [21] proved 
exponential decay to equilibrium in the Maxwellian molecules case $\gamma=0$, and algebraic decay for hard potentials $0<\gamma \leq 1$. Later, the first author 8 proved exponential decay for hard potentials. Toscani and Villani 33 proved algebraic decay for mollified soft potentials $-3<$ $\gamma<0$ (i.e. truncating the singularity of (3) at the origin) excluding the Coulomb case, and the first author [9] proved polynomial convergence for moderately soft potentials $-2<\gamma<0$ and exponential convergence in the case $-1<\gamma<0$. Some results were also obtained in the spatially inhomogeneous case. For potentials in the range $-2 \leq \gamma \leq 1$ and in a close-toequilibrium framework, exponential decay to equilibrium has been established by Mouhot and Neumann [29, Yu [41, and more recently by Tristani, Wu and the first author [10. Still in a perturbative framework and for the Coulomb case, Guo and Strain [31] (see also [30]) proved stretched exponential decay to equilibrium in a high-order Sobolev space with fast decay in the velocity variable. Also, for general initial data and in the Coulomb case, Villani and the second author 22 proved algebraic convergence to equilibrium for (uniformly w.r.t time) a priori smooth solutions.

The aim of this work is to study the large time behaviour of solutions to the spatially homogeneous Landau equation in the Coulomb case. Our proof is based on an entropy-entropy dissipation method.

This method (and its variants) has been widely used to tackle the large time behaviour of many models in kinetic theory (cf. in particular 32, 21, 35, and earlier attempts like [15]) as well as in many other PDEs or integral equations (cf. for example 11 or [17). It is important to emphasize that this method can handle nonlinear equations directly (that is, no linearization is involved).

Roughly speaking, it consists in looking for some Lyapunov functional for the evolution equation (usually called entropy) and then in computing its associated dissipation (usually called entropy dissipation). Then, the existence of functional inequalities relating the entropy dissipation to the entropy itself is investigated. When the method is successful, such inequalities enable to close a differential inequality for the entropy, and yield the large time behaviour.

When the functional inequality involves quantities which grow slowly (that is, polynomially) with respect to time along the flow of the equation, the entropy-entropy dissipation method is said to be "with slowly growing a priori bounds". We refer for example to [33] and [18] for such a situation. In this work, we also use this variant of the entropy-entropy dissipation method.

In kinetic theory, more precisely when Boltzmann and Landau equations are concerned, the functional inequality that hopefully links the entropy dissipation and the entropy was suggested by Cercignani (cf. 11]), and has been known since as Cercignani's conjecture. We refer to [19] for a detailed description of the network of conjectures now bearing this name. We present in this work a variant of the so-called weak Cercignani's conjecture for the Landau equation (with Coulomb potential).

\section{MAin RESUlts}

We state in this section our main results. Hereafter we shall denote polynomial $L^{1}$-moments (for $\ell \in \mathbb{R}$ ) by

$$
M_{\ell}(f):=\|f\|_{L_{\ell}^{1}\left(\mathbb{R}^{3}\right)}:=\int_{\mathbb{R}^{3}}\langle v\rangle^{\ell} f(v) d v, \quad\langle v\rangle:=\left(1+|v|^{2}\right)^{1 / 2},
$$

as well as stretched exponential $L^{1}$-moments (for $s>0, \kappa \in \mathbb{R}$ ) by

$$
M_{s, \kappa}(f):=\|f\|_{L^{1}\left(e^{\kappa\langle v\rangle^{s}} d v\right)}:=\int_{\mathbb{R}^{3}} e^{\kappa\langle v\rangle^{s}} f(v) d v .
$$


Our first main result is a new estimate that bounds from below the entropy dissipation $D(f)$ (defined in (8) ) by a weighted relative Fisher information of $f$ with respect to the associated Maxwellian distribution $\mu$, provided that some higher moment of $f$ is controlled.

Theorem 1. One can find $C:=C(\bar{H})>0$ depending only on $\bar{H}$ such that for all $f \geq 0$ satisfying (the normalization of mass, momentum and energy)

$$
\int_{\mathbb{R}^{3}} f(v) d v=1, \quad \int_{\mathbb{R}^{3}} f(v) v d v=0, \quad \int_{\mathbb{R}^{3}} f(v)|v|^{2} d v=3,
$$

and also satisfying (an upper bound on the entropy)

$$
H(f):=\int_{\mathbb{R}^{3}} f(v) \log f(v) d v \leq \bar{H},
$$

the following inequality holds:

$$
D(f) \geq C(\bar{H})\left(M_{5}(f)\right)^{-1} \int_{\mathbb{R}^{3}} f(v)\left|\frac{\nabla f(v)}{f(v)}+v\right|^{2}\langle v\rangle^{-3} d v .
$$

Remark 1.1. (1) We consider in this work only the case of Coulomb potential, namely $\gamma=$ -3 in the definition of the matrix $a$ given by (3). A straightforward adaptation also gives analogous results for general soft potentials $-4<\gamma<0$. In this situation, estimate (18) becomes

$$
D(f) \geq C(\bar{H})\left(M_{2-\gamma}(f)\right)^{-1} \int_{\mathbb{R}^{3}} f(v)\left|\frac{\nabla f(v)}{f(v)}+v\right|^{2}\langle v\rangle^{\gamma} d v .
$$

(2) We recall that in the case of Maxwell molecules, that is, $\gamma=0$, estimate (19) is already known (cf. [21]), and does not involve any higher moment of $f$ (it involves only $M_{2}(f)$ ).

The proof of this theorem is inspired by the arguments developed by the second author in [14], where it is obtained that the weighted (non relative) Fisher information of $f$ can be bounded from above by the entropy dissipation $D(f)$ plus some constant (depending on the mass and energy of $f$, when $f$ is not normalized). There are nevertheless important differences between the computations of 14 and the proof given here. First, since we allow here the presence in the estimate of a moment of high order, one can use simpler multiplicators than in 14 (no Maxwellian with an arbitrary temperature is introduced in the proof, cf. also [16]). Secondly, and most importantly, one has to keep the exact value of the coefficients appearing in front of linear terms like $v_{i}$, whereas those terms were estimated without too much care in [14].

As a consequence of estimate (18), we shall prove a variant of the so-called weak Cercignani's conjecture for the Landau equation (with Coulomb potential). We refer to [19] for a systematic description of Cercignani's conjecture. Let us say here that the term "weak" means that some quantity other than the mass, energy and (upper bound on the) entropy plays a role in the relationship between $D(f)$ and a weighted version of the relative entropy. Indeed, we need here a control on the fifth moment of $f$ (that is, $M_{5}(f)$ ). This result (variant of the weak Cercignani's conjecture) for the Landau equation (with Coulomb potential) is summarized in the corollary below:

Corollary 1.1. One can find $C:=C(\bar{H})>0$ depending only on $\bar{H}$ such that for all $f \geq 0$ satisfying (the normalization of mass, momentum and energy) (16) and also satisfying (an upper bound on the entropy) (17), the following inequality holds:

$$
D(f) \geq C(\bar{H})\left(M_{5}(f)\right)^{-1} \int\left\{f \log \left(\frac{Z_{1}}{Z_{2}} \frac{f}{\mu}\right)+\frac{Z_{2}}{Z_{1}} \mu-f\right\}\langle v\rangle^{-3} d v,
$$


with $Z_{1}=\int\langle v\rangle^{-3} \mu$ and $Z_{2}=\int\langle v\rangle^{-3} f$. As a consequence, for any $R>0$ (and some absolute constant $C>0$ )

$$
\begin{gathered}
D(f) \geq C(\bar{H})\left(M_{5}(f)\right)^{-1} R^{-3}\left(\int f \log (f / \mu) d v-\int_{\langle v\rangle \geq R} f \log f d v\right. \\
\left.-C \int_{\langle v\rangle \geq R}\langle v\rangle^{2} f d v-C \int_{\langle v\rangle \geq R} \mu d v\right) .
\end{gathered}
$$

Remark 1.2. As already explained in Remark 1.1 (1), this result can be easily adapted to the case of general soft potentials $-4<\gamma<0$, in which case we obtain estimates (20) and (21) replacing $M_{5}(f)$ by $M_{2-\gamma}(f),\langle v\rangle^{-3}$ by $\langle v\rangle^{\gamma}, R^{-3}$ by $R^{\gamma}, Z_{1}$ by $\int\langle v\rangle^{\gamma} \mu$, and $Z_{2}$ by $\int\langle v\rangle^{\gamma} f$.

As an application of the entropy dissipation estimates established in Theorem 1 and Corollary 1.1, we obtain the convergence (with rate) of any ( $H$ - or weak) solution $f$ (of the spatially homogeneous Landau equation with Coulomb potential, and normalized initial data) to the associated Maxwellian equilibrium $\mu$, assuming only that the initial data has finite mass, energy, entropy and some higher $L^{1}$-moment. Before stating our result, let us introduce the notion of solutions that we shall consider in this work.

Definition 1 ( $H$-solutions [36]). Consider a nonnegative $f_{0} \in L_{2}^{1} \cap L \log L\left(\mathbb{R}^{3}\right)$. We say that $f$ is a $H$-solution to the spatially homogeneous Landau equation (1) with Coulomb potential and with initial data $f_{0}$ if it satisfies:

(a) $f \geq 0, f \in C\left([0, \infty) ; \mathcal{D}^{\prime}\left(\mathbb{R}^{3}\right)\right) \cap L^{\infty}\left([0, \infty) ; L_{2}^{1} \cap L \log L\left(\mathbb{R}^{3}\right)\right), f(0)=f_{0}$;

(b) The conservation of mass, momentum and energy, that is, for all $t \geq 0$,

$$
\int f(t, v) \phi(v) d v=\int f_{0}(v) \phi(v) d v \quad \text { for } \quad \phi(v)=1, v_{j},|v|^{2}
$$

(c) The entropy inequality, for all $t \geq 0$,

$$
H(f(t))+\int_{0}^{t} D(f(\tau)) d \tau \leq H\left(f_{0}\right)
$$

(d) $f$ satisfies (11) in the distributional sense: for any test function $\varphi \in C^{1}\left([0, \infty) ; C_{c}^{\infty}\left(\mathbb{R}^{3}\right)\right)$ and for any $t \geq 0$,

$$
\int f(t) \varphi d v-\int f_{0} \varphi(0) d v-\int_{0}^{t} \int f(\tau) \partial_{t} \varphi(\tau) d v d \tau=\int_{0}^{t} \int Q(f, f)(\tau) \varphi(\tau) d v d \tau
$$

where $\int Q(f, f) \varphi d v$ is defined by (5).

Definition 2 (Weak solutions). Consider a nonnegative $f_{0} \in L_{2}^{1} \cap L \log L\left(\mathbb{R}^{3}\right)$. We say that $f$ is a weak solution to the spatially homogeneous Landau equation (1) with Coulomb potential and with initial data $f_{0}$ if it satisfies $(a),(b),(c)$, and $(d)$ of Definition 1 , with the weak formulation of $\int Q(f, f) \varphi d v$ being defined by (6).

As already mentioned, it was proven in $\left[14\right.$ that if $f \in L^{\infty}\left([0, \infty) ; L_{2}^{1} \cap L \log L\left(\mathbb{R}^{3}\right)\right)$ and $D(f) \in L^{1}([0, \infty))$ then $f \in L_{l o c}^{1}\left([0, \infty) ; L_{-3}^{3}\left(\mathbb{R}^{3}\right)\right)$, more precisely estimate (13) holds. Therefore we can replace condition $(a)$ by

$\left(a^{\prime}\right) f \geq 0, f \in C\left([0, \infty) ; \mathcal{D}^{\prime}\left(\mathbb{R}^{3}\right)\right) \cap L^{\infty}\left([0, \infty) ; L_{2}^{1} \cap L \log L\left(\mathbb{R}^{3}\right)\right) \cap L_{\text {loc }}^{1}\left([0, \infty) ; L_{-3}^{3}\left(\mathbb{R}^{3}\right)\right), f(0)=f_{0}$, and then the two notions of solutions are equivalent (because with this new bound we can define $\int Q(f, f) \varphi d v$ by (6) $)$.

Hereafter, in this work, we shall simply say that $f$ is a $H$-or weak solution to the Cauchy problem (11), meaning that $f$ satisfies $\left(a^{\prime}\right)$ (with estimate (13) $),(b),(c)$ and $(d)$, with $\int Q(f, f) \varphi d v$ 
being defined equivalently by (5) or (6). Moreover, we will sometimes split the operator $Q=Q_{1}+Q_{2}$ and use $\int Q_{1}(f, f) \varphi d v$ defined by (5) and $\int Q_{2}(f, f) \varphi d v$ defined by (6).

It is noticed in [36] that (5) and (6) make sense as soon as $f$ satisfies $\left(a^{\prime}\right)$ and $\varphi=\varphi(v) \in$ $W^{2, \infty}\left(\mathbb{R}^{3}\right)$.

We can now state our second main result.

Theorem 2. Let $f_{0} \in L_{2}^{1} \cap L \log L\left(\mathbb{R}^{3}\right)$ satisfy the normalization (12), and consider any global $H$ - or weak solution $f$ to the spatially homogeneous Landau equation (1) with Coulomb potential and with initial data $f_{0}$.

(i) Assume moreover that $f_{0} \in L_{\ell}^{1}\left(\mathbb{R}^{3}\right)$ with $\ell>\frac{19}{2}$. Then for any positive $\beta<\frac{2 \ell^{2}-25 \ell+57}{9(\ell-2)}$, there exists some computable constant $C_{\beta}>0$ depending only on $\beta$, the initial entropy $H\left(f_{0}\right)$ and the initial moment $M_{\ell}\left(f_{0}\right)$, such that

$$
\forall t \geq 0, \quad H(f(t) \mid \mu) \leq C_{\beta}(1+t)^{-\beta} .
$$

(ii) Assume moreover that $f_{0} \in L^{1}\left(\mathbb{R}^{3}, e^{\kappa\langle v\rangle^{s}} d v\right)$, with $\kappa>0$ and $\left.s \in\right] 0,1 / 2[$, or with $\kappa \in$ ] $0,2 / e[$ and $s=1 / 2$. Then there exist some computable constants $C, c>0$ depending only on $\kappa, s$, the initial entropy $H\left(f_{0}\right)$ and the initial moment $M_{s, \kappa}\left(f_{0}\right)$, such that

$$
\forall t \geq 0, \quad H(f(t) \mid \mu) \leq C e^{-c(1+t)^{\frac{s}{3+s}}(\log (1+t))^{-\frac{3}{3+s}}} .
$$

Remark 2.1. (1) The normalization assumption (12) is only for simplicity. The theorem also holds when the initial data are not normalized (i.e. for any $f_{0} \in L_{2}^{1} \cap L \log L\left(\mathbb{R}^{3}\right)$ ), up to the dependence of the constants and to a change in the limiting Maxwellian equilibrium to $\mu_{\rho, u, T}$ defined in (9).

(2) In point (ii), the best rate of convergence towards equilibrium that we can achieve is in the case $s=1 / 2$, where we get a decay with a rate $O\left(e^{-(1+t)^{\frac{1}{7}}(\log (1+t))^{-\frac{6}{7}}}\right)$. We mention that in the close-to-equilibrium regime, the best decay rate is $O\left(e^{-t^{\frac{2}{3}}}\right)$, as can be seen in 31 .

(3) The restriction on the exponent $s \in] 0,1 / 2]$ comes from the results available on the propagation of stretched exponential moments (see Corollary 8.1).

(4) The estimates which are presented in the theorem above concern the relative entropy of the solution of the Landau equation. Thanks to the Cziszar-Kullback-Pinsker inequality (cf. [13, 27), they can be transformed in estimates on the $L^{1}$ norm of $f(t)-\mu$. Then, by interpolation, they also yield estimates for weighted $L^{1}$ norms of $f(t)-\mu$.

(5) As in the case of Theorem 1 (see Remark 1.1), it is possible to extend the estimates of Theorem 2 to the Landau equation with general soft potentials $-4<\gamma<0$. The rates are then modified.

The proof of Theorem 2 uses the entropy dissipation estimate of Theorem 1 (more precisely, that of Corollary 1.1) together with some interpolation inequalities, the regularity estimate (13) and the propagation of $L^{1}$-moments in the Coulomb case (see Lemma 8 and Corollary 8.1).

It is worth mentioning that we do not follow the usual arguments in order to prove Theorem2 2 (see e.g. [33, 9]). Indeed, after obtaining a weak form of Cercignani's conjecture as in (20), one usually obtains, thanks to some interpolation arguments, an inequality of the form

$$
\frac{d}{d t} H(f \mid \mu)=-D(f) \leq-K_{\theta}(f) H(f \mid \mu)^{1+\theta}, \quad \theta>0,
$$

where $K_{\theta}(f)$ is some functional depending on moments and some (high-order) regularity bounds on $f$. Then, in order to close the above differential inequality and conclude thanks to Gronwall's inequality, one needs to prove a priori estimates for solutions $f$ (so that $K_{\theta}(f)$ can be controlled). 
However, when one considers the Coulomb potential, no a priori estimate is known for the highorder regularity of the solutions, the only regularity estimate at hand is indeed (13), that uses again the entropy dissipation $D(f)$. Thus, instead of using an inequality like (22), we shall write a similar inequality, but keeping the exponent 1 instead of $1+\theta$, at the price of some remainder term. We shall then use (13) in order to control part of this remainder term, and only at the very end shall we choose some interpolation (depending on time) in order to close a differential inequality and conclude thanks to some variant of Gronwall's lemma.

\section{EnTROPY DisSIPATION ESTIMATE}

This section is devoted to the proof of Theorem 1, Recall that we have defined in (8), for any $f:=f(v) \geq 0$, by

$$
\begin{gathered}
D(f):=\frac{1}{2} \sum_{i=1}^{3} \sum_{j=1}^{3} \iint_{\mathbb{R}^{3} \times \mathbb{R}^{3}} f(v) f(w)|v-w|^{-1} \Pi_{i j}(v-w)\left(\frac{\partial_{i} f}{f}(v)-\frac{\partial_{i} f}{f}(w)\right) \\
\times\left(\frac{\partial_{j} f}{f}(v)-\frac{\partial_{j} f}{f}(w)\right) d v d w
\end{gathered}
$$

the entropy production of the Landau operator with Coulomb interaction, where $\Pi_{i j}(z)$, defined by (3) is the $i, j$-component of the orthogonal projection $\Pi$ onto $z^{\perp}:=\{y / y \cdot z=0\}$. We also recall the notation $M_{p}(f)$ for the moment of $f$ of order $p$, and, for all $i, j \in\{1,2,3\}$, we define by

$$
P_{i j}^{f}=\int_{\mathbb{R}^{3}} f(v) v_{i} v_{j} d v
$$

the pressure tensor of $f\left(\right.$ when $\left.\int_{\mathbb{R}^{3}} f(v) v d v=0\right)$.

The proof of Theorem 1 is a consequence of the three following Propositions 3 , 4 and 5.

Proposition 3. We denote by $R_{i j}^{f}(v, w)$, for all $i, j \in\{1,2,3\}$, the quantity (i,j-component of the cross product of $v-w$ and $\left.\frac{\nabla f(v)}{f(v)}-\frac{\nabla f(w)}{f(w)}\right)$

$$
R_{i j}^{f}(v, w)=\left(v_{i}-w_{i}\right)\left(\frac{\partial_{j} f(v)}{f(v)}-\frac{\partial_{j} f(w)}{f(w)}\right)-\left(v_{j}-w_{j}\right)\left(\frac{\partial_{i} f(v)}{f(v)}-\frac{\partial_{i} f(w)}{f(w)}\right) .
$$

Then, for all $f:=f(v) \geq 0$ such that (16) is satisfied, and for all $i, j \in\{1,2,3\}, i \neq j$, the following formulas hold:

$$
\begin{aligned}
\frac{\partial_{i} f(v)}{f(v)}= & \frac{v_{j} P_{i j}^{f}+v_{i} P_{i i}^{f}+\int_{\mathbb{R}^{3}} R_{i j}^{f}(v, w) f(w)\left[w_{i} P_{i j}^{f}-w_{j} P_{i i}^{f}\right] d w}{\left(P_{i j}^{f}\right)^{2}-P_{i i}^{f} P_{j j}^{f}}, \\
\frac{\partial_{j} f(v)}{f(v)}= & \frac{v_{i} P_{i j}^{f}+v_{j} P_{j j}^{f}+\int_{\mathbb{R}^{3}} R_{i j}^{f}(v, w) f(w)\left[w_{i} P_{j j}^{f}-w_{j} P_{i j}^{f}\right] d w}{\left(P_{i j}^{f}\right)^{2}-P_{i i}^{f} P_{j j}^{f}}, \\
& v_{i} \frac{\partial_{j} f(v)}{f(v)}-v_{j} \frac{\partial_{i} f(v)}{f(v)}=\int_{\mathbb{R}^{3}} R_{i j}^{f}(v, w) f(w) d w .
\end{aligned}
$$

Note that thanks to the case of equality in Cauchy-Schwarz inequality, we know that $\left(P_{i j}^{f}\right)^{2} \neq$ $P_{i i}^{f} P_{j j}^{f}$, so that formulas (24) and (25) are well defined.

Proposition 3 can be seen as an inversion of formula (23). 
Proof of Proposition 3. We consider $i, j \in\{1,2,3\}$ such that $i \neq j$. Then, we expand $R_{i j}^{f}(v, w)$ in the following way:

$$
\begin{gathered}
R_{i j}^{f}(v, w)=\left[v_{i} \frac{\partial_{j} f(v)}{f(v)}-v_{j} \frac{\partial_{i} f(v)}{f(v)}\right]+w_{j} \frac{\partial_{i} f(v)}{f(v)}-w_{i} \frac{\partial_{j} f(v)}{f(v)} \\
-v_{i} \frac{\partial_{j} f(w)}{f(w)}+v_{j} \frac{\partial_{i} f(w)}{f(w)}+\left[w_{i} \frac{\partial_{j} f(w)}{f(w)}-w_{j} \frac{\partial_{i} f(w)}{f(w)}\right] .
\end{gathered}
$$

Integrating (27) against $f(w) d w$, and recalling conditions (16), we get

$$
\int_{\mathbb{R}^{3}} R_{i j}^{f}(v, w) f(w) d w=v_{i} \frac{\partial_{j} f(v)}{f(v)}-v_{j} \frac{\partial_{i} f(v)}{f(v)},
$$

which is exactly identical to (26).

Integrating then (27) against $f(w) w_{i} d w$, and recalling conditions (16), we get

$$
\int_{\mathbb{R}^{3}} R_{i j}^{f}(v, w) f(w) w_{i} d w=-P_{i i}^{f} \frac{\partial_{j} f(v)}{f(v)}+P_{i j}^{f} \frac{\partial_{i} f(v)}{f(v)}-v_{j}
$$

Finally, integrating (27) against $f(w) w_{j} d w$, and recalling conditions (16) (or exchanging $i$ and $j$ in (28), we get

$$
\int_{\mathbb{R}^{3}} R_{i j}^{f}(v, w) f(w) w_{j} d w=-P_{i j}^{f} \frac{\partial_{j} f(v)}{f(v)}+P_{j j}^{f} \frac{\partial_{i} f(v)}{f(v)}+v_{i} .
$$

Considering (28), (29) as a $2 \times 2$ linear system with unknowns $\frac{\partial_{i} f(v)}{f(v)}, \frac{\partial_{j} f(v)}{f(v)}$, we get thanks to Cramer's formulas (recalling that $\left(P_{i j}^{f}\right)^{2} \neq P_{i i}^{f} P_{j j}^{f}$ because of the case of equality in CauchySchwarz inequality)

$$
\begin{aligned}
& \frac{\partial_{i} f(v)}{f(v)}=\frac{\operatorname{Det}\left(\begin{array}{cc}
v_{j}+\int_{\mathbb{R}^{3}} R_{i j}^{f}(v, w) f(w) w_{i} d w & -P_{i i}^{f} \\
-v_{i}+\int_{\mathbb{R}^{3}} R_{i j}^{f}(v, w) f(w) w_{j} d w & -P_{i j}^{f}
\end{array}\right)}{\operatorname{Det}\left(\begin{array}{ll}
P_{i j}^{f} & -P_{i i}^{f} \\
P_{j j}^{f} & -P_{i j}^{f}
\end{array}\right)}, \\
& \frac{\partial_{j} f(v)}{f(v)}=\frac{\operatorname{Det}\left(\begin{array}{cc}
P_{i j}^{f} & v_{j}+\int_{\mathbb{R}^{3}} R_{i j}^{f}(v, w) f(w) w_{i} d w \\
P_{j j}^{f} & -v_{i}+\int_{\mathbb{R}^{3}} R_{i j}^{f}(v, w) f(w) w_{j} d w
\end{array}\right)}{\operatorname{Det}\left(\begin{array}{ll}
P_{i j}^{f} & -P_{i i}^{f} \\
P_{j j}^{f} & -P_{i j}^{f}
\end{array}\right)},
\end{aligned}
$$

which is exactly identical to formulas (24), (25).

Proposition 4. We now define

$$
\Delta_{f}:=\inf _{i, j=1,2,3 ; i \neq j}\left(P_{i i}^{f} P_{j j}^{f}-\left(P_{i j}^{f}\right)^{2}\right) .
$$

Then there exists $C>0$ an explicitly computable constant number such that for all $f:=f(v) \geq 0$ satisfying (16),

$$
\begin{gathered}
\int_{\mathbb{R}^{3}} f(v)\left|\frac{\nabla f(v)}{f(v)}+v\right|^{2}\langle v\rangle^{-3} d v \\
\leq C \Delta_{f}^{-2}\left(\sup _{i, j=1,2,3 ; i \neq j}\left(P_{i j}^{f}\right)^{2}+\sup _{j=1,2,3}\left|P_{j j}^{f}-1\right|^{2}+M_{5}(f) D(f)\right) .
\end{gathered}
$$


Proof of Proposition 屜. Thanks to (24), we see that (for any $i, j \in\{1,2,3\}$ such that $i \neq j$ )

$$
\begin{gathered}
\frac{\partial_{i} f(v)}{f(v)}+v_{i}=v_{j} \frac{P_{i j}^{f}}{\left(P_{i j}^{f}\right)^{2}-P_{i i}^{f} P_{j j}^{f}}+v_{i}\left(1+\frac{P_{i i}^{f}}{\left(P_{i j}^{f}\right)^{2}-P_{i i}^{f} P_{j j}^{f}}\right) \\
+\frac{\int_{\mathbb{R}^{3}} R_{i j}^{f}(v, w) f(w)\left[w_{i} P_{i j}^{f}-w_{j} P_{i i}^{f}\right] d w}{\left(P_{i j}^{f}\right)^{2}-P_{i i}^{f} P_{j j}^{f}},
\end{gathered}
$$

so that, remembering that $P_{i j}^{f} \leq 3 / 2$ for all $i, j \in\{1,2,3\}, i \neq j$, and $P_{i i}^{f} \leq 3$ for all $i \in\{1,2,3\}$, since $\sum_{i=1}^{3} P_{i i}^{f}=3$,

$$
\begin{gathered}
\left|\frac{\partial_{i} f(v)}{f(v)}+v_{i}\right|^{2} \leq 3 \Delta_{f}^{-2}\left(\left|v_{j}\right|^{2}\left(P_{i j}^{f}\right)^{2}+\left|v_{i}\right|^{2}\left|\left(P_{i j}^{f}\right)^{2}+P_{i i}^{f}\left(1-P_{j j}^{f}\right)\right|^{2}\right. \\
\left.+\left|\int_{\mathbb{R}^{3}} R_{i j}^{f}(v, w) f(w)\left[w_{i} P_{i j}^{f}-w_{j} P_{i i}^{f}\right] d w\right|^{2}\right) \\
\leq 3 \Delta_{f}^{-2}\left(\left|v_{j}\right|^{2}\left(P_{i j}^{f}\right)^{2}+2\left|v_{i}\right|^{2}\left(P_{i j}^{f}\right)^{4}+18\left|v_{i}\right|^{2}\left(1-P_{j j}^{f}\right)^{2}\right. \\
\left.+9\left[\int_{\mathbb{R}^{3}}\left|R_{i j}^{f}(v, w)\right| f(w)\left(\left|w_{i}\right|+\left|w_{j}\right|\right) d w\right]^{2}\right) .
\end{gathered}
$$

Then,

$$
\begin{aligned}
\int_{\mathbb{R}^{3}} f(v)\left|\frac{\partial_{i} f(v)}{f(v)}+v_{i}\right|^{2}\langle v\rangle^{-3} d v \leq 3 \Delta_{f}^{-2}\left(\frac{11}{2}\left(P_{i j}^{f}\right)^{2}+18\left(1-P_{j j}^{f}\right)^{2}\right. \\
+9 \int_{\mathbb{R}^{3}}\langle v\rangle^{-3} f(v)\left\{\int_{\mathbb{R}^{3}}\left|R_{i j}^{f}(v, w)\right|^{2} f(w)|v-w|^{-3} d w\right\} \\
\left.\times\left\{\int_{\mathbb{R}^{3}} f(w)|v-w|^{3}\left(\left|w_{i}\right|+\left|w_{j}\right|\right)^{2} d w\right\} d v\right) \\
\leq \Delta_{f}^{-2}\left(\frac{33}{2}\left(P_{i j}^{f}\right)^{2}+54\left(1-P_{j j}^{f}\right)^{2}+27 \int_{\mathbb{R}^{3}} \int_{\mathbb{R}^{3}} f(v) f(w)\left|R_{i j}^{f}(v, w)\right|^{2}|v-w|^{-3} d w d v\right. \\
\left.\times \sup _{v \in \mathbb{R}^{3}}\langle v\rangle^{-3} \int_{\mathbb{R}^{3}} f(w)\left(4|v|^{3}+4|w|^{3}\right) 2|w|^{2} d w\right) .
\end{aligned}
$$

Observing then (cf. 14, p.11-12) that

$$
D(f)=\frac{1}{4} \sum_{i=1}^{3} \sum_{j=1}^{3} \int_{\mathbb{R}^{3}} \int_{\mathbb{R}^{3}} f(v) f(w)\left|R_{i j}^{f}(v, w)\right|^{2}|v-w|^{-3} d w d v,
$$

we end up with the estimate

$$
\begin{aligned}
\int_{\mathbb{R}^{3}} f(v) & \left|\frac{\nabla f(v)}{f(v)}+v\right|^{2}\langle v\rangle^{-3} d v \leq \Delta_{f}^{-2}\left(\frac{99}{2} \sup _{i, j \in\{1,2,3\}, i \neq j}\left(P_{i j}^{f}\right)^{2}\right. \\
& \left.+162 \sup _{j \in\{1,2,3\}}\left(1-P_{j j}^{f}\right)^{2}+3456 M_{5}(f) D(f)\right),
\end{aligned}
$$

so that (31) holds with $C=3456$. 
Proposition 5. One can find $C:=C(\bar{H})$ depending only on $\bar{H}$ such that for all $f \geq 0$ satisfying (16), (17), the following inequalities hold:

$$
\begin{gathered}
\Delta_{f} \geq C(\bar{H}), \\
\sup _{i, j \in\{1,2,3\}, i \neq j}\left(P_{i j}^{f}\right)^{2} \leq C(\bar{H}) M_{5}(f) D(f), \\
\sup _{i, j \in\{1,2,3\}, i \neq j}\left|P_{i i}^{f}-P_{j j}^{f}\right|^{2} \leq C(\bar{H}) M_{5}(f) D(f) .
\end{gathered}
$$

Proof of Proposition 5. We first observe that (thanks to [14, p.15), for any $\delta>0$,

$$
\begin{gathered}
\Delta_{f}:=\inf _{i, j=1,2,3 ; i \neq j}\left(P_{i i}^{f} P_{j j}^{f}-\left(P_{i j}^{f}\right)^{2}\right) \\
\geq \inf _{|\theta| \leq 1} \inf _{k=i, j ; l=i, j ; k \neq l}\left(\int\left|v_{k}-\theta v_{l}\right|^{2} f(v) d v\right)^{2} \\
\geq \delta^{4} \inf _{|\theta| \leq 1} \inf _{k=i, j ; l=i, j ; k \neq l}\left(\int_{\left|v_{k}-\theta v_{l}\right| \geq \delta,|v| \leq \sqrt{6}} f(v) d v\right)^{2} \\
\geq \delta^{4}\left(1-\sup _{|\theta| \leq 1} \sup _{k=i, j ; l=i, j ; k \neq l} \int_{\left|v_{k}-\theta v_{l}\right| \leq \delta,|v| \leq \sqrt{6}} f(v) d v-\int_{|v| \geq \sqrt{6}} f(v) d v\right)^{2} \\
\geq \delta^{4}\left(\frac{1}{2}-\sup _{|\theta| \leq 1} \sup _{k=i, j ; l=i, j ; k \neq l} \int_{\frac{\left|v_{k}-\theta v_{l}\right|}{\sqrt{1+\theta^{2}}} \leq \frac{\delta}{\sqrt{1+\theta^{2}}},|v| \leq \sqrt{6}} f(v) d v\right)^{2} \\
\geq \delta^{4}\left(\frac{1}{2}-\sup _{|\theta| \leq 1} \sup _{|A| \leq 48 \frac{\delta}{\sqrt{1+\theta^{2}}}} \int_{A} f(v) d v\right)^{2} .
\end{gathered}
$$

Using now the estimate (for all $q>0$ and $M>1$ )

$$
\sup _{|A| \leq q} \int_{A} f(v) d v \leq M q+\frac{\bar{H}}{\log M}
$$

we see that

$$
\Delta_{f} \geq \delta^{4}\left(\frac{1}{2}-48 \delta M-\frac{\bar{H}}{\log M}\right)^{2},
$$

so that taking $M=e^{4 \bar{H}}$ and $48 \delta M=\frac{1}{8}$ (that is $\delta=2^{-7} 3^{-1} e^{-4 \bar{H}}$ ), we end up with

$$
\Delta_{f} \geq \frac{1}{2^{34}} \frac{1}{3^{4}} e^{-16 \bar{H}},
$$

and estimate (32) is proven.

We now turn to the proof of estimates (33) and (34).

Inserting (24) and (25) in (26), we see that

$$
\begin{gathered}
\left(v_{i}^{2}-v_{j}^{2}\right) P_{i j}^{f}+v_{i} v_{j}\left(P_{j j}^{f}-P_{i i}^{f}\right) \\
+\int_{\mathbb{R}^{3}} R_{i j}^{f}(v, w) f(w)\left[v_{i} w_{i} P_{j j}^{f}-v_{i} w_{j} P_{i j}^{f}-v_{j} w_{i} P_{i j}^{f}+v_{j} w_{j} P_{i i}^{f}\right] d w \\
=\left[\left(P_{i j}^{f}\right)^{2}-P_{i i}^{f} P_{j j}^{f}\right] \int_{\mathbb{R}^{3}} R_{i j}^{f}(v, w) f(w) d w
\end{gathered}
$$

and writing

$$
\left|v_{i} w_{i} P_{j j}^{f}-v_{i} w_{j} P_{i j}^{f}-v_{j} w_{i} P_{i j}^{f}+v_{j} w_{j} P_{i i}^{f}\right| \leq 9|v||w|
$$


it follows

$$
\left|\left(v_{i}^{2}-v_{j}^{2}\right) P_{i j}^{f}+v_{i} v_{j}\left(P_{j j}^{f}-P_{i i}^{f}\right)\right| \leq \int_{\mathbb{R}^{3}}\left|R_{i j}^{f}(v, w)\right| f(w)[9+9|v||w|] d w .
$$

Then

$$
\begin{gathered}
\int_{\mathbb{R}^{3}} f(v)\langle v\rangle^{-5}\left|\left(v_{i}^{2}-v_{j}^{2}\right) P_{i j}^{f}+v_{i} v_{j}\left(P_{j j}^{f}-P_{i i}^{f}\right)\right|^{2} d v \\
\leq \int_{\mathbb{R}^{3}} f(v)\langle v\rangle^{-5}\left|\int_{\mathbb{R}^{3}}\right| R_{i j}^{f}(v, w)|f(w)[9+9|v||w|] d w|^{2} d v \\
\leq \int_{\mathbb{R}^{3}} f(v)\langle v\rangle^{-5}\left\{\int_{\mathbb{R}^{3}}\left|R_{i j}^{f}(v, w)\right|^{2} f(w)|v-w|^{-3} d w\right\} \\
\quad \times\left\{\int_{\mathbb{R}^{3}} f(w)|v-w|^{3}[9+9|v||w|]^{2} d w\right\} d v \\
\leq C D(f) \sup _{v \in \mathbb{R}^{3}}\langle v\rangle^{-5} \int_{\mathbb{R}^{3}} f(w)\left(|v|^{3}+|w|^{3}\right)\left(1+|v|^{2}|w|^{2}\right) d w \\
\leq C D(f) \sup _{v \in \mathbb{R}^{3}}\langle v\rangle^{-5} \int_{\mathbb{R}^{3}} f(w)\left(|v|^{3}+|v|^{5}|w|^{2}+|w|^{3}+|v|^{2}|w|^{5}\right) d w \\
\leq C D(f) M_{5}(f),
\end{gathered}
$$

where $C>0$ is a (computable) constant number.

We now observe that

where

$$
\begin{gathered}
\int_{\mathbb{R}^{3}} f(v)\langle v\rangle^{-5}\left|\left(v_{i}^{2}-v_{j}^{2}\right) P_{i j}^{f}+v_{i} v_{j}\left(P_{j j}^{f}-P_{i i}^{f}\right)\right|^{2} d v \\
\geq S_{f}\left[\left(P_{i j}^{f}\right)^{2}+\frac{1}{4}\left(P_{j j}^{f}-P_{i i}^{f}\right)^{2}\right]
\end{gathered}
$$

$$
S_{f}:=\inf _{\phi \in \mathbb{R}} \int_{\mathbb{R}^{3}} f(v)\langle v\rangle^{-5}\left|\left(v_{i}^{2}-v_{j}^{2}\right) \cos \phi+2 v_{i} v_{j} \sin \phi\right|^{2} d v .
$$

Introducing cylindrical coordinates defined by $v_{i}=r \cos \theta, v_{j}=r \sin \theta$, and $v_{k}=z$ (where $k \neq i$ and $k \neq j$ ), we see that for all $\varepsilon>0$ (and assuming without loss of generality that $i=1$, $j=2, k=3)$

$$
\begin{aligned}
& S_{f}=\inf _{\phi \in \mathbb{R}} \int_{z \in \mathbb{R}} \int_{\theta=0}^{2 \pi} \int_{r \in \mathbb{R}_{+}} f(r \cos \theta, r \sin \theta, z)\left(1+r^{2}+|z|^{2}\right)^{-5 / 2} r^{4}|\cos (2 \theta-\phi)|^{2} r d r d \theta d z \\
& \geq|\sin \varepsilon|^{2}\left[\int_{z \in \mathbb{R}} \int_{\theta=0}^{2 \pi} \int_{r \in \mathbb{R}_{+}} f(r \cos \theta, r \sin \theta, z) r^{4}\left(1+r^{2}+|z|^{2}\right)^{-5 / 2} r d r d \theta d z\right. \\
&\left.\quad-\sup _{\phi \in \mathbb{R}} \int_{z \in \mathbb{R}} \int_{\theta=0}^{2 \pi} \int_{r \in \mathbb{R}_{+}} f(r \cos \theta, r \sin \theta, z) \mathbf{1}_{\left\{\left|2 \theta-\phi-\frac{\pi}{2} \mathbb{Z}\right| \leq \varepsilon\right\}} r d r d \theta d z\right] .
\end{aligned}
$$

As a consequence, denoting by $|\cdot|$ the Lebesgue measure on $\mathbb{R}^{3}$, for all $\varepsilon, \delta, R_{1}, R_{2}>0$,

$$
\begin{gathered}
S_{f} \geq|\sin \varepsilon|^{2}\left[\int_{z \in \mathbb{R}} \int_{\theta=0}^{2 \pi} \int_{r \in \mathbb{R}_{+}} f(r \cos \theta, r \sin \theta, z)\right. \\
\times\left(1+r^{2}+|z|^{2}\right)^{-5 / 2} r^{4} \mathbf{1}_{\{r \geq \delta\}} \mathbf{1}_{\left\{r^{2}+|z|^{2} \leq R_{1}^{2}\right\}} r d r d \theta d z \\
-\sup _{\phi \in \mathbb{R}} \int_{z \in \mathbb{R}} \int_{\theta=0}^{2 \pi} \int_{r \in \mathbb{R}_{+}} f(r \cos \theta, r \sin \theta, z) \mathbf{1}_{\left\{r^{2}+|z|^{2} \leq R_{2}^{2}\right\}} \mathbf{1}_{\left\{\mid 2 \theta-\phi-\frac{\pi}{2} \mathbb{Z}\right\} \mid \leq \varepsilon} r d r d \theta d z \\
\left.-\int_{z \in \mathbb{R}} \int_{\theta=0}^{2 \pi} \int_{r \in \mathbb{R}_{+}} f(r \cos \theta, r \sin \theta, z) \mathbf{1}_{\left\{r^{2}+|z|^{2} \geq R_{2}^{2}\right\}} r d r d \theta d z\right]
\end{gathered}
$$




$$
\begin{gathered}
\geq|\sin \varepsilon|^{2}\left[\frac { \delta ^ { 4 } } { ( 1 + R _ { 1 } ^ { 2 } ) ^ { 5 / 2 } } \left(1-\int_{v \in \mathbb{R}^{3}} f(v) \mathbf{1}_{\left\{\left|v_{1}\right|^{2}+\left|v_{2}\right|^{2} \leq \delta^{2}\right\}} \mathbf{1}_{\left\{|v| \leq R_{1}\right\}} d v\right.\right. \\
\left.\left.-\int_{v \in \mathbb{R}^{3}} f(v) \mathbf{1}_{\left\{|v| \geq R_{1}\right\}} d v\right)-\int_{v \in \mathbb{R}^{3}} f(v) \mathbf{1}_{\left\{|v| \geq R_{2}\right\}} d v-\sup _{|A| \leq 16 R_{2}^{3} \varepsilon} \int_{A} f(v) d v\right] \\
\geq|\sin \varepsilon|^{2}\left[\frac{\delta^{4}}{\left(1+R_{1}^{2}\right)^{5 / 2}}\left(1-\sup _{|A| \leq 2 \pi R_{1} \delta^{2}} \int_{A} f(v) d v-\frac{3}{R_{1}^{2}}\right)\right. \\
\left.-\frac{3}{R_{2}^{2}}-\sup _{|A| \leq 16 R_{2}^{3} \varepsilon} \int_{A} f(v) d v\right] .
\end{gathered}
$$

Using now estimate (35), we see that for all $\varepsilon, \delta, R_{1}, R_{2}, M_{1}, M_{2}>0$,

$$
S_{f} \geq|\sin \varepsilon|^{2}\left[\frac{\delta^{4}}{\left(1+R_{1}^{2}\right)^{5 / 2}}\left(1-2 \pi M_{1} R_{1} \delta^{2}-\frac{\bar{H}}{\log M_{1}}-\frac{3}{R_{1}^{2}}\right)-\frac{3}{R_{2}^{2}}-16 M_{2} R_{2}^{3} \varepsilon-\frac{\bar{H}}{\log M_{2}}\right] .
$$

The proof of (33), (34) is concluded by selecting successively $R_{1}$ large enough, $M_{1}$ large enough, $\delta>0$ small enough, $R_{2}$ large enough, $M_{2}$ large enough, and $\varepsilon>0$ small enough.

For example, selecting $R_{1}, M_{1}$ and $\delta$ in such a way that

$$
\frac{3}{R_{1}^{2}}=\frac{\bar{H}}{\ln M_{1}}=2 \pi M_{1} R_{1} \delta^{2}=\frac{1}{4}
$$

we end up with

$$
S_{f} \geq|\sin \varepsilon|^{2}\left[\frac{13^{-5 / 2}}{48} \frac{e^{-8 \bar{H}}}{64 \pi^{2}}-\frac{3}{R_{2}^{2}}-16 M_{2} R_{2}^{3} \varepsilon-\frac{\bar{H}}{\log M_{2}}\right]
$$

Then, we fix $R_{2}, M_{2}$ and $\varepsilon$ in such a way that

$$
\frac{3}{R_{2}^{2}}=\frac{\bar{H}}{\log M_{2}}=16 M_{2} R_{2}^{2} \varepsilon=\frac{13^{-5 / 2}}{48} \frac{e^{-8 \bar{H}}}{256 \pi^{2}}
$$

We can now conclude the proof of Theorem 1 .

Proof of Theorem 11. Thanks to Proposition 4 and Proposition 5, we see that it only remains to estimate $\sup _{j=1,2,3}\left|P_{j j}^{f}-1\right|$ in terms of $\sup _{i, j \in\{1,2,3\}, i \neq j}\left|P_{i i}^{f}-P_{j j}^{f}\right|$.

In order to do so, we use the identity $\sum_{j=1}^{3} P_{j j}^{f}=3$. We observe that

$$
\left|P_{11}^{f}-1\right|=\left|P_{11}^{f}-\frac{1}{3} \sum_{j=1}^{3} P_{j j}^{f}\right| \leq \frac{1}{3}\left|P_{11}^{f}-P_{22}^{f}\right|+\frac{1}{3}\left|P_{11}^{f}-P_{33}^{f}\right| .
$$

Doing the same computation with $P_{22}^{f}, P_{33}^{f}$, we end up with

$$
\sup _{j=1,2,3}\left|P_{j j}^{f}-1\right| \leq \frac{2}{3} \sup _{i, j \in\{1,2,3\}, i \neq j}\left|P_{i i}^{f}-P_{j j}^{f}\right|
$$

and Theorem 1 is proven.

We conclude this section with the proof of Corollary 1.1 . 
Proof of Corollary 1.1. Let $f \geq 0$ satisfy the normalization (16), and define the weighted (with weight $\langle v\rangle^{\alpha}$ for $\alpha \in \mathbb{R}$ ) relative Fisher information of $f$ with respect to $\mu$ (the centred reduced Gaussian) by

$$
I_{\alpha}(f \mid \mu):=\int_{\mathbb{R}^{3}} \frac{|\nabla(f / \mu)|^{2}}{(f / \mu)}\langle v\rangle^{\alpha} d \mu(v)=\int_{\mathbb{R}^{3}} f\left|\frac{\nabla f}{f}+v\right|^{2}\langle v\rangle^{\alpha} d v .
$$

With this notation, Theorem 1 becomes

$$
D(f) \geq C(\bar{H}) M_{5}(f)^{-1} I_{-3}(f \mid \mu) .
$$

We define $\nu(v):=Z_{1}^{-1}\langle v\rangle^{-3} \mu(v)$ and $g(v):=Z_{2}^{-1}\langle v\rangle^{-3} f(v)$, where $Z_{1}:=\int\langle v\rangle^{-3} d \mu(v)$ and $Z_{2}:=\int\langle v\rangle^{-3} f(v) d v$ are normalization constants so that $\int \nu(v) d v=\int g(v) d v=1$. Therefore we can rewrite

$$
I_{-3}(f \mid \mu)=\int \frac{|\nabla(f / \mu)|^{2}}{(f / \mu)}\langle v\rangle^{-3} d \mu(v)=Z_{2} \int \frac{|\nabla(g / \nu)|^{2}}{(g / \nu)} d \nu(v)=: Z_{2} I(g \mid \nu),
$$

where $I(g \mid \nu):=I_{0}(g \mid \nu)$ denotes the standard (i.e. without weight) relative Fisher information of $g$ with respect to $\nu$. We now observe that $d \nu(v)=e^{-U(v)} d v$, with $U(v)=\frac{1}{2}|v|^{2}+\frac{3}{2} \log (1+$ $\left.|v|^{2}\right)+U_{0}$, and where $U_{0}:=\log \left((2 \pi)^{3 / 2} Z_{1}\right)$ is a normalization constant. We easily compute

$$
\text { Hess } U(v)=I d+\frac{3}{\langle v\rangle^{2}} I d-\frac{6}{\langle v\rangle^{4}} v \otimes v \text {. }
$$

Hence we obtain (for all $\xi, v \in \mathbb{R}^{3}$ ),

$$
\langle\operatorname{Hess} U(v) \xi, \xi\rangle=\left(1+\frac{3}{\langle v\rangle^{2}}\right)|\xi|^{2}-\frac{6}{\langle v\rangle^{4}}(\xi \cdot v)^{2} \geq\left(1+\frac{3}{\langle v\rangle^{2}}-\frac{6|v|^{2}}{\langle v\rangle^{4}}\right)|\xi|^{2}>\frac{5}{8}|\xi|^{2} .
$$

Indeed, for all $z \in \mathbb{R}_{+}\left(z=|v|^{2}\right)$,

$$
1+\frac{3}{1+z}-\frac{6 z}{(1+z)^{2}}=\frac{z^{2}-z+4}{(1+z)^{2}} \geq \frac{5}{8} .
$$

The probability measure $d \nu$ satisfies then a log-Sobolev inequality thanks to the Bakry-Émery criterion (see [7, 5]). Therefore, for some $C>0$,

$$
I(g \mid \nu) \geq C \int \frac{g}{\nu} \log \frac{g}{\nu} d \nu(v)=C \int\left\{\frac{g}{\nu} \log \frac{g}{\nu}+1-\frac{g}{\nu}\right\} d \nu(v) .
$$

Thanks to estimate (36), we finally deduce, for some (new) constant $C(\bar{H})>0$,

$$
D(f) \geq C(\bar{H})\left(M_{5}(f)\right)^{-1} \int\left\{f \log \left(\frac{Z_{1}}{Z_{2}} \frac{f}{\mu}\right)+\frac{Z_{2}}{Z_{1}} \mu-f\right\}\langle v\rangle^{-3} d v,
$$

and the proof of (20) is complete. Now, for any $R>0$, we estimate the integral from below remembering that $x \log x+1-x \geq 0$ for $x>0$,

$$
\begin{gathered}
D(f) \geq C(\bar{H})\left(M_{5}(f)\right)^{-1} R^{-3} \int_{\langle v\rangle \leq R}\left\{f \log \left(\frac{Z_{1}}{Z_{2}} \frac{f}{\mu}\right)+\frac{Z_{2}}{Z_{1}} \mu-f\right\} d v \\
\geq C(\bar{H})\left(M_{5}(f)\right)^{-1} R^{-3}\left(\int_{\langle v\rangle \leq R} f \log (f / \mu) d v+\log \left(Z_{1} / Z_{2}\right) \int_{\langle v\rangle \leq R} f d v\right. \\
\left.+\int_{\langle v\rangle \leq R}(\mu-f) d v+\left(Z_{2} / Z_{1}-1\right) \int_{\langle v\rangle \leq R} \mu d v\right) .
\end{gathered}
$$




$$
\begin{gathered}
\geq C(\bar{H})\left(M_{5}(f)\right)^{-1} R^{-3}\left(\int f \log (f / \mu) d v+\frac{Z_{2}}{Z_{1}}\left(\frac{Z_{1}}{Z_{2}} \log \left(Z_{1} / Z_{2}\right)+1-\frac{Z_{1}}{Z_{2}}\right)\right. \\
\quad-\int_{\langle v\rangle \geq R} f \log (f / \mu) d v+\log \left(Z_{2} / Z_{1}\right) \int_{\langle v\rangle \geq R} f d v \\
\left.\quad+\int_{\langle v\rangle \geq R}(f-\mu) d v+\left(1-Z_{2} / Z_{1}\right) \int_{\langle v\rangle \geq R} \mu d v\right) \\
\geq C(\bar{H})\left(M_{5}(f)\right)^{-1} R^{-3}\left(\int f \log (f / \mu) d v-\int_{\langle v\rangle \geq R} f \log (f / \mu) d v\right. \\
\left.\quad+\left(1+\log \left(Z_{2} / Z_{1}\right)\right) \int_{\langle v\rangle \geq R} f d v-\left(Z_{2} / Z_{1}\right) \int_{\langle v\rangle \geq R} \mu d v\right) .
\end{gathered}
$$

Since $\int f=\int \mu=1$ and $\int\langle v\rangle^{2} f=\int\langle v\rangle^{2} \mu=4$, we easily obtain that $2^{-11 / 2} \leq Z_{1}, Z_{2} \leq 1$. Then

$$
\begin{gathered}
D(f) \geq C(\bar{H})\left(M_{5}(f)\right)^{-1} R^{-3}\left(\int f \log (f / \mu) d v-\int_{\langle v\rangle \geq R} f \log f d v\right. \\
\left.-C \int_{\langle v\rangle \geq R}\langle v\rangle^{2} f d v-C \int_{\langle v\rangle \geq R} \mu d v\right),
\end{gathered}
$$

which completes the proof of (21).

\section{Moments estimates}

In this section we prove estimates for the polynomial and exponential $L^{1}$-moments defined in (14) and (15). For the sake of completeness we shall consider, only in this section, the Landau operator $Q$ (see (2) ) for general soft potentials, i.e. a matrix $a_{i j}$ (see (3)) for the whole range of soft potentials $-4<\gamma<0$. It is worth mentioning that, in the case $-2 \leq \gamma \leq 0$, estimates for polynomial moments have been established in [38, 40, 9] and stretched exponential moments in 9]. Moreover, in the case $-4<\gamma<-2$ polynomial moments estimates have been established in [39, 14. We shall improve the above mentioned results in Lemma 7. Corollary 7.1] Lemma 8 and Corollary 8.1

We begin with a key lemma on the coercivity of the collision operator in weighted $L^{1}$-space.

Lemma 6. Assume $-4<\gamma<0$. Let $f$ be a nonnegative function and $\chi$ be either $\mathbf{1}_{|\cdot| \leq 1}$, or a smooth $C_{c}^{\infty}(\mathbb{R})$ radially symmetric cutoff function that satisfies $\mathbf{1}_{B_{1 / 2}} \leq \chi \leq \mathbf{1}_{B_{1}}$. Let $\chi_{\eta}(\cdot)=\chi(\cdot / \eta)$ with $\left.\left.\eta \in\right] 0,1\right], l>2$ and

$$
I=\iint_{\mathbb{R}^{3} \times \mathbb{R}^{3}} f(v) f(w)|v-w|^{\gamma} \chi_{\eta}^{c}(v-w)\langle v\rangle^{l-2}\left\{-\langle v\rangle^{2}+\langle w\rangle^{2}\right\} d w d v,
$$

where $\chi_{\eta}^{c}=1-\chi_{\eta}$.

Then there exist constants $K, C>0$ such that

$$
\begin{aligned}
I \leq & -K M_{0}(f)^{1-\gamma / 2} M_{2}(f)^{\gamma / 2} M_{l+\gamma}(f)+C M_{2}(f) M_{l-2+\gamma}(f) \\
& +C\left(M_{2}(f) / M_{0}(f)\right)^{l / 2-1+\gamma} M_{0}(f) M_{2}(f) .
\end{aligned}
$$

Proof. We decompose the integral into two parts $I=I_{1}+I_{2}$ with

$$
\begin{aligned}
& I_{1}=\iint_{\{|v-w|<|w|\} \cap\{|v-w|<|v|\}} f(v) f(w)|v-w|^{\gamma} \chi_{\eta}^{c}(v-w)\langle v\rangle^{l-2}\left\{-\langle v\rangle^{2}+\langle w\rangle^{2}\right\} d w d v, \\
& I_{2}=\iint_{\{|v-w| \geq|w|\} \cup\{|v-w| \geq|v|\}} f(v) f(w)|v-w|^{\gamma} \chi_{\eta}^{c}(v-w)\langle v\rangle^{l-2}\left\{-\langle v\rangle^{2}+\langle w\rangle^{2}\right\} d w d v .
\end{aligned}
$$


For the first term $I_{1}$, we easily get $I_{1} \leq 0$ thanks to Young's inequality and using the symmetry of the region $\{|v-w|<|w|\} \cap\{|v-w|<|v|\}$ :

$$
\begin{aligned}
& \iint_{\{|v-w|<|w|\} \cap\{|v-w|<|v|\}} f(v) f(w)|v-w|^{\gamma} \chi_{\eta}^{c}(v-w)\langle v\rangle^{l-2}\langle w\rangle^{2} d w d v \\
& \quad \leq \iint_{\{|v-w|<|w|\} \cap\{|v-w|<|v|\}} f(v) f(w)|v-w|^{\gamma} \chi_{\eta}^{c}(v-w)\left[\frac{l-2}{l}\langle v\rangle^{l}+\frac{2}{l}\langle w\rangle^{l}\right] d w d v \\
& \quad=\iint_{\{|v-w|<|w|\} \cap\{|v-w|<|v|\}} f(v) f(w)|v-w|^{\gamma} \chi_{\eta}^{c}(v-w)\langle v\rangle^{l} d w d v .
\end{aligned}
$$

Next we observe that

$$
\begin{aligned}
I_{2}= & \iint_{\{|v-w| \geq|w|\} \cup\{|v-w| \geq|v|\}} f(v) f(w)|v-w|^{\gamma}\left(\chi_{\eta}^{c}(v-w)-\mathbf{1}_{|v-w| \geq 1}\right)\langle v\rangle^{l-2}\left\{-\langle v\rangle^{2}+\langle w\rangle^{2}\right\} d w d v \\
& -\iint_{\{|v-w| \geq|w|\} \cup\{|v-w| \geq|v|\}} f(v) f(w)|v-w|^{\gamma} \mathbf{1}_{|v-w| \geq 1}\langle v\rangle^{l-2}\left\{\langle v\rangle^{2}-\langle w\rangle^{2}\right\} d w d v .
\end{aligned}
$$

Using the estimate $\mathbf{1}_{|v-w| \geq 1} \leq \mathbf{1}_{|v-w| \geq \eta} \leq \chi_{\eta}^{c}(v-w)$ since $\left.\left.\eta \in\right] 0,1\right]$ and following an argument similar to the one used for $I_{1}$, we obtain that the first term in the right-hand side of the previous identity is nonpositive. Hence we have

$$
I_{2} \leq-\iint_{\{|v-w| \geq|w|\} \cup\{|v-w| \geq|v|\}} f(v) f(w)|v-w|^{\gamma} \mathbf{1}_{|v-w| \geq 1}\langle v\rangle^{l-2}\left\{\langle v\rangle^{2}-\langle w\rangle^{2}\right\} d w d v=: A+B,
$$

and we estimate each term separately.

For the term $A$, we first write that

$$
A \leq-\iint_{\{|v-w| \geq|w|\}} f(v) f(w)|v-w|^{\gamma} \mathbf{1}_{|v-w| \geq 1}\langle v\rangle^{l} d w d v
$$

and then we notice that the region $\{|v-w| \geq|w|\} \cap\{|v-w| \geq 1\}$ contains $\{|v| \geq 2 r\} \cap\{|w| \leq r\}$ for any $r \geq 1$. Therefore, using that $-|v-w|^{\gamma} \leq-C\langle w\rangle^{\gamma}\langle v\rangle^{\gamma}$,

$$
\begin{aligned}
A & \leq-C \iint_{\{|v| \geq 2 r\} \cap\{|w| \leq r\}} f(v) f(w)|v-w|^{\gamma}\langle v\rangle^{l} d w d v \\
& \leq-C\left(\int_{\{|w| \leq r\}}\langle w\rangle^{\gamma} f(w) d w\right)\left(\int_{\{|v| \geq 2 r\}}\langle v\rangle^{l+\gamma} f(v) d v\right) .
\end{aligned}
$$

We can easily compute

$$
\begin{aligned}
\int_{\{|w| \leq r\}}\langle w\rangle^{\gamma} f(w) d w & \geq\langle r\rangle^{\gamma} \int_{|w| \leq r} f(w) d w \\
& =\langle r\rangle^{\gamma}\left(M_{0}(f)-\int_{\{|w| \geq r\}} f(w) d w\right) \geq\langle r\rangle^{\gamma}\left(M_{0}(f)-\frac{M_{2}(f)}{\langle r\rangle^{2}}\right),
\end{aligned}
$$

and also

$$
\int_{\{|v| \geq 2 r\}}\langle v\rangle^{l+\gamma} f(v) d v=M_{l+\gamma}(f)-\int_{\{|v| \leq 2 r\}}\langle v\rangle^{l+\gamma} f(v) d v \geq M_{l+\gamma}(f)-\langle 2 r\rangle^{l-2+\gamma} M_{2}(f) .
$$

Gathering the previous estimates, we get

$$
\begin{gathered}
A \leq C\left(\frac{M_{2}(f)}{\langle r\rangle^{2}}-M_{0}(f)\right)\langle r\rangle^{\gamma} M_{l+\gamma}(f)+C\langle 2 r\rangle^{l-2+2 \gamma} M_{0}(f) M_{2}(f) \\
\leq-K M_{0}(f)^{1-\gamma / 2} M_{2}(f)^{\gamma / 2} M_{l+\gamma}(f)+C\left(M_{2}(f) / M_{0}(f)\right)^{l / 2-1+\gamma} M_{0}(f) M_{2}(f)
\end{gathered}
$$

by choosing $r$ such that $M_{2}(f) /\langle r\rangle^{2}=M_{0}(f) / 2$. 
For the term $B$, we first decompose it into $B=B_{1}+B_{2}+B_{3}$, with

$$
\begin{aligned}
& B_{1}:=\iint_{\{|v-w| \geq|v|\} \cap\{|v-w| \geq 1\}} f(v) f(w)|v-w|^{\gamma}\langle v\rangle^{l-2}\langle w\rangle^{2} d w d v, \\
& B_{2}:=\iint_{\{|w| \leq|v-w| \leq 2|w|\} \cap\{|v-w| \geq 1\}} f(v) f(w)|v-w|^{\gamma}\langle v\rangle^{l-2}\langle w\rangle^{2} d w d v, \\
& B_{3}:=\iint_{\{|v-w| \geq 2|w|\} \cap\{|v-w| \geq 1\}} f(v) f(w)|v-w|^{\gamma}\langle v\rangle^{l-2}\langle w\rangle^{2} d w d v,
\end{aligned}
$$

and we claim that

$$
B_{j} \leq C M_{2}(f) M_{l-2+\gamma}(f), \quad j=1,2,3 .
$$

Indeed, we first remark that for all the terms, we have $|v-w|^{\gamma} \leq C\langle v-w\rangle^{\gamma}$ since $|v-w| \geq 1$, thus in order to prove (38), we only need to prove that $|v-w| \geq c|v|$ for some constant $c>0$ in each case $j=1,2,3$. The first case $j=1$ is immediate since $|v-w| \geq|v|$. We then observe that $|w| \leq|v-w| \leq 2|w|$ implies $|v-w| \sim|w|$ and also $|v| \leq C|w|$, which completes the case $j=2$. Finally, when $|v-w| \geq 2|w|$, we obtain $|v-w| \sim|v|$ and the case $j=3$ also holds.

We get the desired result by patching together estimates (37) and (38).

We first state and prove estimates for $L^{1}$-moments in the moderately soft potentials case $-2 \leq \gamma<0$. We improve the results of [38, 40, 9].

Lemma 7. Assume that $-2 \leq \gamma<0$. Let $f_{0} \in L_{2}^{1} \cap L \log L$ and consider any global $H-$ or weak solution $f$ to the spatially homogeneous Landau equation (1) with initial data $f_{0}$. Suppose further that $f_{0} \in L_{l}^{1}$ for some $l>2$. Then, there exists a constant $C>0$ depending on $\gamma$, $M_{0}\left(f_{0}\right), M_{2}\left(f_{0}\right)$ (but not on l) such that, for all $t \geq 0$,

$$
M_{l}(f(t)) \leq M_{l}(f(0))+C l^{\frac{l+\gamma}{2}} t .
$$

Proof. For simplicity we only give here the a priori estimates for the moments. The rigorous proof for any solution follows the same arguments as the ones that we shall present in Step 2 of the proof of Lemma 8 below, in the case of very soft potentials.

Recall that thanks to the conservation of mass and energy, we have $M_{0}(f(t))=M_{0}\left(f_{0}\right)$ and $M_{2}(f(t))=M_{2}\left(f_{0}\right)$ for all $t \geq 0$. The equation for moments is (see e.g. 20])

$$
\frac{d}{d t} M_{l}(f)=\iint f(v) f(w)|v-w|^{\gamma}\langle v\rangle^{l}\left\{-2 l+2 l\langle v\rangle^{-2}\langle w\rangle^{2}+l(l-2)\langle v\rangle^{-4}\left[|v|^{2}|w|^{2}-(v \cdot w)^{2}\right]\right\} d w d v .
$$

Because of the singularity of $|v-w|^{\gamma}$, we split it into two parts $|v-w|^{\gamma} \mathbf{1}_{|v-w| \geq 1}$ and $\mid v-$ $\left.w\right|^{\gamma} \mathbf{1}_{|v-w| \leq 1}$, and we denote respectively $T_{1}$ and $T_{2}$ each term associated.

For the term $T_{2}$, we write

$$
\begin{aligned}
T_{2}= & l \iint_{\mathbb{R}^{6}}|v-w|^{\gamma} \mathbf{1}_{|v-w| \leq 1}\langle v\rangle^{l-2}\left\{-2\langle v\rangle^{2}+2\langle w\rangle^{2}\right\} f(v) f(w) d w d v \\
& +l(l-2) \iint_{\mathbb{R}^{6}}|v-w|^{\gamma} \mathbf{1}_{|v-w| \leq 1}\langle v\rangle^{l-4}\left\{|v|^{2}|w|^{2}-(v \cdot w)^{2}\right\} f(v) f(w) d w d v \\
= & : T_{21}+T_{22} .
\end{aligned}
$$

Using Young's inequality, we easily obtain

$$
\begin{array}{r}
\iint_{\mathbb{R}^{6}} f(v) f(w)|v-w|^{\gamma} \mathbf{1}_{|v-w| \leq 1}\langle v\rangle^{l-2}\langle w\rangle^{2} d w d v \\
\leq \iint_{\mathbb{R}^{6}} f(v) f(w)|v-w|^{\gamma} \mathbf{1}_{|v-w| \leq 1}\langle v\rangle^{l} d w d v
\end{array}
$$


and this implies $T_{21} \leq 0$. Moreover, using the inequality $|v|^{2}|w|^{2}-(v \cdot w)^{2} \leq|w|^{2}|v-w|^{2}$, we get, since $\gamma+2 \geq 0$,

$$
\begin{aligned}
T_{22} & \leq C l^{2} \iint_{\mathbb{R}^{6}} f(v) f(w)|v-w|^{\gamma+2} \mathbf{1}_{|v-w| \leq 1}\langle v\rangle^{l-4}\langle w\rangle^{2} d w d v \\
& \leq C l^{2} M_{2}(f) M_{l-4}(f) .
\end{aligned}
$$

We now investigate the term $T_{1}$, that we write

$$
\begin{aligned}
T_{1}= & -2 l \iint_{\mathbb{R}^{6}}|v-w|^{\gamma} \mathbf{1}_{|v-w| \geq 1}\langle v\rangle^{l-2}\left\{\langle v\rangle^{2}-\langle w\rangle^{2}\right\} f(v) f(w) d w d v \\
& +l(l-2) \iint_{\mathbb{R}^{6}}|v-w|^{\gamma} \mathbf{1}_{|v-w| \geq 1}\langle v\rangle^{l-4}\left\{|v|^{2}|w|^{2}-(v \cdot w)^{2}\right\} f(v) f(w) d w d v \\
= & I+I I .
\end{aligned}
$$

Thanks to Lemma 6 (with $\chi=\mathbf{1}_{|\cdot| \leq 1}$ ), we already have

$$
\begin{aligned}
I \leq & -K l M_{0}(f)^{1-\gamma / 2} M_{2}(f)^{\gamma / 2} M_{l+\gamma}(f)+C l M_{2}(f) M_{l-2+\gamma}(f) \\
& +C l\left(M_{2}(f) / M_{0}(f)\right)^{l / 2-1+\gamma} M_{0}(f) M_{2}(f) .
\end{aligned}
$$

We consider now the term $I I$. If $l \leq 4$, we easily observe that

$$
I I \leq C l^{2}\left(M_{2}(f)\right)^{2} .
$$

Now let $l>4$. We split $I I=I I_{1}+I I_{2}$, with

$$
I I_{1}=l(l-2) \iint_{\{|v-w| \geq 1\} \cap\{|w| \leq|v|\}}|v-w|^{\gamma}\langle v\rangle^{l-4}\left\{|v|^{2}|w|^{2}-(v \cdot w)^{2}\right\} f(v) f(w) d w d v,
$$

and

$$
I I_{2}=l(l-2) \iint_{\{|v-w| \geq 1\} \cap\{|w| \geq|v|\}}|v-w|^{\gamma}\langle v\rangle^{l-4}\left\{|v|^{2}|w|^{2}-(v \cdot w)^{2}\right\} f(v) f(w) d w d v .
$$

Using the estimate $|v|^{2}|w|^{2}-(v \cdot w)^{2} \leq|w|^{2}|v-w|^{2}$, we get

$$
\begin{aligned}
I I_{1} & \leq C l^{2} \iint_{\{|v-w| \geq 1\} \cap\{|w| \leq|v|\}}|v-w|^{\gamma+2}\langle v\rangle^{l-4}\langle w\rangle^{2} f(v) f(w) d w d v \\
& \leq C l^{2} \iint_{\{|v-w| \geq 1\} \cap\{|w| \leq|v|\}}\langle v\rangle^{l-2+\gamma}\langle w\rangle^{2} f(v) f(w) d w d v \\
& \leq C l^{2} M_{2}(f) M_{l-2+\gamma}(f) .
\end{aligned}
$$

Using now the inequality $|v|^{2}|w|^{2}-(v \cdot w)^{2} \leq|v|^{2}|v-w|^{2}$, it follows that

$$
\begin{aligned}
I I_{2} & \leq C l^{2} \iint_{\{|v-w| \geq 1\} \cap\{|w| \geq|v|\}}|v-w|^{\gamma+2}\langle v\rangle^{l-2} f(v) f(w) d w d v \\
& \leq C l^{2} \iint_{\{|v-w| \geq 1\} \cap\{|w| \geq|v|\}}\langle v\rangle^{l-2}\langle w\rangle^{\gamma+2} f(v) f(w) d w d v \\
& \leq C l^{2} \iint_{\{|v-w| \geq 1\} \cap\{|w| \geq|v|\}}\langle v\rangle^{2}\langle w\rangle^{l-2+\gamma} f(v) f(w) d w d v \\
& \leq C l^{2} M_{2}(f) M_{l-2+\gamma}(f) .
\end{aligned}
$$

Gathering the previous estimates and recalling that $M_{0}(f)$ and $M_{2}(f)$ are constants, we get, for constants $K, C>0$,

$$
\begin{aligned}
\frac{d}{d t} M_{l}(f) & \leq-K l M_{l+\gamma}(f)+C l^{2} M_{l-4}(f)+C\left(l+l^{2} \mathbf{1}_{l>4}\right) M_{l-2+\gamma}(f)+C\left(l+l^{2} \mathbf{1}_{l \leq 4}\right) \\
& \leq-K l M_{l+\gamma}(f)+C l^{2} M_{l-2+\gamma}(f)+C l^{2}
\end{aligned}
$$


since $M_{l-4}(f) \leq M_{l-2+\gamma}(f)$ (remember that $-2 \leq \gamma<0$ ). If $l \leq 4-\gamma$, then $M_{l-2+\gamma}(f(t))$ is uniformly bounded and we easily get

$$
M_{l}(f(t)) \leq M_{l}\left(f_{0}\right)+C t .
$$

Consider now $l>4-\gamma$. Thanks to Young's inequality, for any $\epsilon>0$,

$$
M_{l-2+\gamma}(f) \leq M_{2}^{\frac{2}{l-2+\gamma}}(f) M_{l+\gamma}^{\frac{l-4+\gamma}{l-2+\gamma}}(f) \leq C \epsilon^{-\frac{l-4+\gamma}{2}} M_{2}(f)+\epsilon M_{l+\gamma}(f) .
$$

Hence it yields

$$
\frac{d}{d t} M_{l}(f)+K l M_{l+\gamma}(f) \leq C l^{2} \epsilon M_{l+\gamma}(f)+C l^{2} \epsilon^{-\frac{l-4+\gamma}{2}}+C l .
$$

Choosing $\epsilon=\frac{K}{2 C} l^{-1}$, we get

$$
\frac{d}{d t} M_{l}(f)+\frac{K}{2} l M_{l+\gamma}(f) \leq C\left(l^{\frac{l+\gamma}{2}}+C l\right) \leq C l^{\frac{l+\gamma}{2}},
$$

from which we deduce

$$
M_{l}(t) \leq M_{l}\left(f_{0}\right)+C l^{\frac{l+\gamma}{2}} t,
$$

which completes the proof.

Corollary 7.1. Consider the same setting as in Lemma 7. Suppose further that $M_{s, \kappa}\left(f_{0}\right)=$ $\int f_{0}(v) e^{\kappa\langle v\rangle^{s}} d v<\infty$ with $\kappa>0$ and $0<s<2$, or with $0<\kappa<1 /(2 e)$ and $s=2$.

Then, for some constant $C>0$ depending only on the parameters $\gamma, s, \kappa$ and the initial mass and energy (that is, depending on $M_{0}\left(f_{0}\right), M_{2}\left(f_{0}\right)$ ),

(1) If $s+\gamma<0$, for all $t \geq 0$,

$$
M_{s, \kappa}(f(t)) \leq M_{s, \kappa}\left(f_{0}\right)+C t .
$$

(2) If $s+\gamma \geq 0$, for all $t \geq 0$,

$$
M_{s, \kappa}(f(t)) \leq M_{s, \kappa}\left(f_{0}\right)+C .
$$

Remark 7.1. As a direct consequence of Corollary 7.1 (2), the exponential convergence to equilibrium established in [9, Theorem 1.4], for the case $-1<\gamma<0$, can be extended to the case $-2 \leq \gamma \leq-1$, as explained in [9, Remark 1.5].

Proof. (1) We write $e^{\kappa\langle v\rangle^{s}}=\sum_{j=0}^{\infty} \frac{\kappa^{j}}{j !}\langle v\rangle^{j s}$ so that

$$
M_{s, \kappa}(f(t))=\int\left(\sum_{j=0}^{\infty} \frac{\kappa^{j}}{j !}\langle v\rangle^{j s}\right) f(t) d v=\sum_{j=0}^{\infty} \int \frac{\kappa^{j}}{j !}\langle v\rangle^{j s} f(t) d v=\sum_{j=0}^{\infty} \frac{\kappa^{j}}{j !} M_{j s}(f(t)),
$$

where we have used Tonelli's theorem since the integrand in nonnegative (for any solution $f$ ). Thanks to Lemma 7 we therefore obtain

$$
M_{s, \kappa}(f(t)) \leq C t \sum_{j=0}^{\infty} \frac{\kappa^{j}}{j !}(s j)^{\frac{s j}{2}+\frac{\gamma}{2}}+M_{s, \kappa}\left(f_{0}\right),
$$

and we only need to prove that the sum is finite. We rewrite

$$
\beta_{j}:=\frac{\kappa^{j}}{j !}(s j)^{\frac{j s}{2}+\frac{\gamma}{2}}=\left(\kappa s^{\frac{s}{2}}\right)^{j}(s j)^{\frac{\gamma}{2}} \frac{j^{\frac{s j}{2}}}{j !}, \quad j ! \sim(j / e)^{j} \sqrt{2 \pi j} \quad \text { as } \quad j \rightarrow \infty,
$$

hence we easily obtain that $\sum_{j=1}^{\infty} \beta_{j}<\infty$ for any $\kappa>0$ if $s<2$, or for $0<\kappa<1 /(2 e)$ if $s=2$. 
(2) As in the proof of Lemma [7, we only give here the a priori estimates. Coming back to (39), one has

$$
\begin{aligned}
\frac{d}{d t} M_{s, \kappa}(f) & =\sum_{j=0}^{\infty} \frac{\kappa^{j}}{j !} \frac{d}{d t} M_{j s}(f) \\
& \leq \sum_{j=0}^{\infty} \frac{\kappa^{j}}{j !}\left\{-K j s M_{j s+\gamma}(f)+C(j s)^{\frac{j s}{2}+\frac{\gamma}{2}}\right\} \\
& \leq-K \kappa s \sum_{j=1}^{\infty} \frac{\kappa^{j-1}}{(j-1) !} M_{j s+\gamma}(f)+C \sum_{j=0}^{\infty} \frac{\kappa^{j}}{j !}(j s)^{\frac{j s}{2}+\gamma / 2} \\
& =-K \kappa s \sum_{n=0}^{\infty} \frac{\kappa^{n}}{n !} M_{n s+s+\gamma}(f)+C \sum_{j=0}^{\infty} \frac{\kappa^{j}}{j !}(j s)^{\frac{j s}{2}+\gamma / 2}=: I+I I .
\end{aligned}
$$

Since $s+\gamma \geq 0$, we know that $-M_{n s+s+\gamma}(f) \leq-M_{n s}(f)$, which implies the estimate $I \leq$ $-K \kappa s M_{s, \kappa}(f)$. The second term $I I$ is finite for any $\kappa>0$ if $s<2$, or for $0<\kappa<1 /(2 e)$ if $s=2$. We finally obtain

$$
\frac{d}{d t} M_{s, \kappa}(f) \leq-K \kappa s M_{s, \kappa}(f)+C
$$

which implies the desired uniform in time bound.

We now investigate the case of very soft potentials $-4<\gamma \leq-2$. We get new estimates on the propagation of the moments which improve the results of [39, Appendix B, p. 193] and [14].

Lemma 8. Assume that $-4<\gamma \leq-2$. Let $f_{0} \in L_{2}^{1} \cap L \log L\left(\mathbb{R}^{3}\right)$ and consider any global $H$ - or weak solution $f$ to the spatially homogeneous Landau equation (1) with initial data $f_{0}$. Assume moreover that $f_{0} \in L_{l}^{1}$ for some $l>2$. Then there exists a constant $C=$ $C\left(\gamma, M_{0}\left(f_{0}\right), M_{2}\left(f_{0}\right), H\left(f_{0}\right)\right)>0$ (that does not depend on l) such that

$$
M_{l}(f(t)) \leq C M_{l}\left(f_{0}\right)+C l^{(l-6) \frac{|\gamma+1|}{\gamma+4}-\gamma} t .
$$

Proof. We divide the proof into two steps.

Step 1: A priori estimates. We follow the argument of [39, Appendix B, p. 193] that uses the entropy formulation of solutions (cf. [36]). Let $\eta=c l^{-1}$ and $\chi_{\eta}(\cdot)=\chi(\cdot / \eta)$ where $\left.c \in\right] 0,1 / 2[$ is a (small) constant and $\chi \in C_{c}^{\infty}(\mathbb{R})$ is a smooth radially symmetric cutoff function such that $\mathbf{1}_{B_{1 / 2}} \leq \chi \leq \mathbf{1}_{B_{1}}$, as in Lemma 6. Recall that $a(z)=|z|^{\gamma+2} \Pi(z)$ and decompose $a=a_{\eta}+a_{\eta}^{c}$ with $a_{\eta}(z):=\chi_{\eta}(z)|z|^{\gamma+2} \Pi(z)$ and $a_{\eta}^{c}(z):=\chi_{\eta}^{c}(z)|z|^{\gamma+2} \Pi(z)$, where $\chi_{\eta}^{c}(z):=1-\chi_{\eta}(z)$. We then write

$$
\frac{d}{d t} M_{l}(f)=I+I I
$$

where

$$
\begin{gathered}
I=-\frac{1}{2} \iint f(v) f(w) \chi_{\eta}|v-w|^{\gamma+2} \Pi\left(\frac{\nabla f}{f}(v)-\frac{\nabla f}{f}(w)\right)(\nabla \varphi(v)-\nabla \varphi(w)) d w d v, \\
I I=-\frac{1}{2} \iint f(v) f(w) \chi_{\eta}^{c}|v-w|^{\gamma+2} \Pi\left(\frac{\nabla f}{f}(v)-\frac{\nabla f}{f}(w)\right)(\nabla \varphi(v)-\nabla \varphi(w)) d w d v,
\end{gathered}
$$

with $\Pi=\Pi(v-w), \chi_{\eta}=\chi_{\eta}(v-w)$ and $\varphi(v)=\langle v\rangle^{l}$. 
Therefore, by Cauchy-Schwarz inequality and using that $\chi_{\eta}^{2}(v-w) \leq \mathbf{1}_{|v-w| \leq \eta}$, it follows

$$
\begin{aligned}
|I| \leq & \left(\iint f(v) f(w)|v-w|^{\gamma+2} \Pi\left(\frac{\nabla f}{f}(v)-\frac{\nabla f}{f}(w)\right)\left(\frac{\nabla f}{f}(v)-\frac{\nabla f}{f}(w)\right) d w d v\right)^{1 / 2} \\
& \times\left(\iint f(v) f(w)|v-w|^{\gamma+2} \mathbf{1}_{|v-w| \leq \eta} \Pi(\nabla \varphi(v)-\nabla \varphi(w))(\nabla \varphi(v)-\nabla \varphi(w)) d w d v\right)^{1 / 2},
\end{aligned}
$$

and the first integral is bounded by $\sqrt{2 D(f)}$, see (8). For $\varphi(v)=\langle v\rangle^{l}$, we have

$$
|v-w|^{2} \Pi(\nabla \varphi(v)-\nabla \varphi(w))(\nabla \varphi(v)-\nabla \varphi(w))=l^{2}\left[|v|^{2}|w|^{2}-(v \cdot w)^{2}\right]\left[\langle v\rangle^{l-2}-\langle w\rangle^{l-2}\right]^{2} .
$$

Using the estimate

$$
|v|^{2}|w|^{2}-(v \cdot w)^{2} \leq \min \left(|v|^{2},|w|^{2}\right)|v-w|^{2}
$$

and $\left|\langle v\rangle^{l-2}-\langle w\rangle^{l-2}\right| \leq l \max \left(\langle v\rangle^{l-3},\langle w\rangle^{l-3}\right)|v-w|$, we finally get

$$
|v-w|^{2} \Pi(\nabla \varphi(v)-\nabla \varphi(w))(\nabla \varphi(v)-\nabla \varphi(w)) \leq C l^{4}\left\{\langle v\rangle^{2 l-4}+\langle w\rangle^{2 l-4}\right\}|v-w|^{4} .
$$

Then

$$
|I| \leq C l^{2} D(f)^{1 / 2}\left(\iint f(v) f(w)|v-w|^{\gamma+4} \mathbf{1}_{|v-w| \leq \eta}\langle v\rangle^{2 l-4} d w d v\right)^{1 / 2} .
$$

Since the last integral is over $\left\{|v-w| \leq \eta=c l^{-1}\right\}$, we claim that there exist universal constants $C_{i}(i=1,2)$ such that $C_{1}\langle v\rangle^{l} \leq\langle w\rangle^{l} \leq C_{2}\langle v\rangle^{l}$. Indeed, we have

$$
\begin{aligned}
\langle v\rangle^{l}= & \left(1+|v|^{2}\right)^{l / 2} \leq\left(\langle w\rangle^{2}+2 c l^{-1}\left(|w|+c l^{-1}\right)\right)^{l / 2} \\
\leq & \sum_{k=0}^{[(l+2) / 4]} \frac{\frac{l}{2}\left(\frac{l}{2}-1\right) \ldots\left(\frac{l}{2}-k+1\right)}{k !}\left[\langle w\rangle^{2 k}\left(2 c l^{-1}\right)^{l / 2-k}\left(|w|+c l^{-1}\right)^{l / 2-k}\right. \\
& \left.+\left(2 c l^{-1}\right)^{k}\left(|w|+c l^{-1}\right)^{k}\langle w\rangle^{l-2 k}\right] \\
\leq & \sum_{k=0}^{[(l+2) / 4]} \frac{1}{k !}\langle w\rangle^{l} \leq C\langle w\rangle^{l},
\end{aligned}
$$

where we use the fractional binomial expansion. Using the symmetry of $v$ and $w$, we conclude the proof of the claim. Now we obtain

$$
\begin{aligned}
|I| & \leq C l^{2} D(f)^{1 / 2}\left(\iint f(v) f(w)|v-w|^{\gamma+4} \mathbf{1}_{|v-w| \leq \eta}\langle w\rangle^{l-4}\langle v\rangle^{l} d w d v\right)^{1 / 2} \\
& \leq C l^{2} D(f)^{1 / 2} \eta^{\gamma / 2+2} M_{l}^{1 / 2}(f) M_{l-4}^{1 / 2}(f) \\
& \leq D(f) M_{l}(f)+C l^{-\gamma} M_{l-4}(f) .
\end{aligned}
$$

For the term $I I$, we use the usual weak formulation (cf. [36]), obtained from (41) by performing an integration by parts w.r.t. both $v$ and $w$ :

$$
I I=\iint f(v) f(w)\left\{a_{\eta}^{c}(v-w): \nabla^{2} \varphi(v)+2 b_{\eta}^{c}(v-w) \cdot \nabla \varphi(v)\right\} d w d v,
$$

where $\left(b_{\eta}^{c}(z)\right)_{i=1,2,3}=\sum_{j=1}^{3} \partial_{j}\left(a_{\eta}^{c}(z)\right)_{i j}=\chi_{\eta}^{c}(|z|) b_{i}(z)+\sum_{j=1}^{3}\left(\partial_{j}\left[\chi_{\eta}^{c}(|z|)\right]\right) a_{i j}(z)$. It follows then

$$
\begin{aligned}
I I= & \iint f(v) f(w) \chi_{\eta}^{c}(|v-w|)\left\{a(v-w): \nabla^{2} \varphi(v)+2 b(v-w) \cdot \nabla \varphi(v)\right\} d w d v \\
& +2 \sum_{i=1}^{3} \sum_{j=1}^{3} \iint f(v) f(w)\left(\partial_{j}\left[\chi_{\eta}^{c}(|v-w|)\right]\right) a_{i j}(v-w) \partial_{i} \varphi(v) d w d v,
\end{aligned}
$$


and we remark that the second integral vanishes since, for $j \in\{1,2,3\},\left(\partial_{j}\left[\chi_{\eta}^{c}(|z|)\right]\right)=\left(\chi_{\eta}^{c}\right)^{\prime}(|z|)|z|^{-1} z_{j}$ and $\sum_{j=1}^{3} a_{i j}(z) z_{j}=0$. We finally obtain (see the proof of Lemma 7 )

$$
\begin{aligned}
I I= & 2 l \iint f(v) f(w)|v-w|^{\gamma} \chi_{\eta}^{c}(v-w)\langle v\rangle^{l-2}\left\{-\langle v\rangle^{2}+\langle w\rangle^{2}\right\} d w d v \\
& +l(l-2) \iint f(v) f(w)|v-w|^{\gamma} \chi_{\eta}^{c}(v-w)\langle v\rangle^{l-4}\left\{|v|^{2}|w|^{2}-(v \cdot w)^{2}\right\} d w d v \\
= & I I_{1}+I I_{2} .
\end{aligned}
$$

Using the elementary inequality $|v|^{2}|w|^{2}-(v \cdot w)^{2} \leq|w|^{2}|v-w|^{2}$, we easily obtain, thanks to the estimate $\chi_{\eta}^{c}(v-w) \leq \mathbf{1}_{|v-w| \geq \eta / 2}$,

$$
I I_{2} \leq l^{2} \iint_{\mathbb{R}^{6}} f(v) f(w)|v-w|^{\gamma+2} \mathbf{1}_{|v-w| \geq \eta / 2}\langle w\rangle^{2}\langle v\rangle^{l-4} d w d v \leq C l^{-\gamma} M_{2}(f) M_{l-4}(f) .
$$

The term $I I_{1}$ is controlled thanks to Lemma 6 (with $\chi$ a $C^{\infty}$ function), which gives

$$
\begin{aligned}
I I_{1} \leq & -K l M_{0}(f)^{1-\gamma / 2} M_{2}(f)^{\gamma / 2} M_{l+\gamma}(f)+C l M_{2}(f) M_{l-2+\gamma}(f) \\
& +C l\left(M_{2}(f) / M_{0}(f)\right)^{l / 2-1+\gamma} M_{0}(f) M_{2}(f) .
\end{aligned}
$$

Finally, gathering estimates (43), (44) and (45), and using that $M_{0}(f)$ and $M_{2}(f)$ are constant in time, it follows

$$
\frac{d}{d t} M_{l}(f)+K l M_{l+\gamma}(f) \leq D(f) M_{l}(f)+C l^{-\gamma} M_{l-4}(f)+C l M_{l-2+\gamma}(f)+C l .
$$

Notice that $M_{l-2+\gamma}(f) \leq M_{l-4}(f)$ because $-4<\gamma \leq-2$, thus the third term in the right-hand side of (46) can be absorbed into the second one. We also recall that $\int_{0}^{\infty} D(f(s)) d s \leq C<\infty$ for some positive constant $C$ depending on $H\left(f_{0}\right)$.

If $2<l \leq 6$, then $\sup _{t \geq 0} M_{l-4}(f(t)) \leq C$, hence

$$
\frac{d}{d t} M_{l}(f)+K l M_{l+\gamma}(f) \leq D(f) M_{l}(f)+C,
$$

and by Gronwall's lemma,

$$
M_{l}(f(t)) \leq C M_{l}\left(f_{0}\right)+C t .
$$

Suppose now that $l>6$. We use Young's inequality to obtain, for any $\epsilon>0$,

$$
M_{l-4}(f) \leq C \epsilon^{-\frac{l-6}{\gamma+4}} M_{2}(f)+\epsilon M_{l+\gamma}(f) .
$$

Coming back to (46) and choosing $\epsilon=\frac{K}{2 C} l^{1+\gamma}$, we then get

$$
\begin{aligned}
\frac{d}{d t} M_{l}(f)+\frac{K}{2} l M_{l+\gamma}(f) & \leq D(f) M_{l}(f)+C l^{(l-6) \frac{|\gamma+1|}{\gamma+4}-\gamma}+C l \\
& \leq D(f) M_{l}(f)+C l^{(l-6) \frac{|\gamma+1|}{\gamma+4}-\gamma},
\end{aligned}
$$

hence

$$
M_{l}(f(t)) \leq C M_{l}\left(f_{0}\right)+C l^{(l-6) \frac{|\gamma+1|}{\gamma+4}-\gamma} t,
$$

which yields the desired result.

Step 2: Rigorous proof. Let $W_{\delta}^{l}(v)=\langle v\rangle^{l}\left(1+\delta|v|^{2}\right)^{-\frac{l}{2}}$ with $\left.\delta \in\right] 0,1 / 2[$ being a (small) parameter, and set $M_{l}^{\delta}(f)=\int f(v) W_{\delta}^{l}(v) d v$. It follows that $W_{\delta}^{l} \in W^{2, \infty}\left(\mathbb{R}^{3}\right)$ and then it can be chosen as a test function in the formulation of the weak solution, that is,

$$
\int_{\mathbb{R}^{3}} f(t) W_{\delta}^{l} d v=\int_{\mathbb{R}^{3}} f_{0} W_{\delta}^{l} d v+\int_{0}^{t} \int_{\mathbb{R}^{3}} Q(f, f) W_{\delta}^{l} d v d t .
$$


Similarly to (40) and (41), we have

$$
\int_{\mathbb{R}^{3}} Q(f, f) W_{\delta}^{l} d v=I_{\delta}+I I_{\delta}
$$

where

$$
I_{\delta}=-\frac{1}{2} \iint f(v) f(w) \chi_{\eta}|v-w|^{\gamma+2} \Pi\left(\frac{\nabla f}{f}(v)-\frac{\nabla f}{f}(w)\right)\left(\nabla W_{\delta}^{l}(v)-\nabla W_{\delta}^{l}(w)\right) d w d v,
$$

and

$$
\begin{aligned}
I I_{\delta} & =-\frac{1}{2} \iint f(v) f(w) \chi_{\eta}^{c}|v-w|^{\gamma+2} \Pi\left(\frac{\nabla f}{f}(v)-\frac{\nabla f}{f}(w)\right)\left(\nabla W_{\delta}^{l}(v)-\nabla W_{\delta}^{l}(w)\right) d w d v \\
(48) & =\iint f(v) f(w) \chi_{\eta}^{c}\left(a(v-w): \nabla^{2} W_{\delta}^{l}(v)+2 b(v-w) \cdot \nabla W_{\delta}^{l}(v)\right) d w d v
\end{aligned}
$$

with $\Pi=\Pi(v-w)$ and $\chi_{\eta}=\chi_{\eta}(v-w)$.

Estimate of $I_{\delta}$. By following the estimate of $I$ in Step 1, we first have

$$
\begin{aligned}
\left|I_{\delta}\right| \leq & \left(\iint f(v) f(w)|v-w|^{\gamma+2} \Pi\left(\frac{\nabla f}{f}(v)-\frac{\nabla f}{f}(w)\right)\left(\frac{\nabla f}{f}(v)-\frac{\nabla f}{f}(w)\right) d w d v\right)^{1 / 2} \\
& \times\left(\iint f(v) f(w)|v-w|^{\gamma+2} \chi_{\eta} \Pi\left(\nabla W_{\delta}^{l}(v)-\nabla W_{\delta}^{l}(w)\right)\left(\nabla W_{\delta}^{l}(v)-\nabla W_{\delta}^{l}(w)\right) d w d v\right)^{1 / 2} .
\end{aligned}
$$

We claim that

$$
\left|I_{\delta}\right| \leq C l^{2} \sqrt{2 D(f)}\left(\iint f(v) f(w) \chi_{\eta}|v-w|^{\gamma+4} W_{\delta}^{l}(v) W_{\delta}^{l}(w)\langle w\rangle^{-4} d w d v\right)^{\frac{1}{2}} .
$$

Indeed, following the computation of (42), we first have

$$
1_{|v-w| \leq c l^{-1}}\left(1+\delta|v|^{2}\right)^{\frac{l}{2}} \sim 1_{|v-w| \leq c l^{-1}}\left(1+\delta|w|^{2}\right)^{\frac{l}{2}},
$$

from which, together with the fact $1_{|v-w| \leq c l^{-1}}\langle v\rangle^{l} \sim 1_{|v-w| \leq c l^{-1}}\langle w\rangle^{l}$, we get the (uniform w.r.t. $\delta \in] 0,1 / 2[$ ) estimate

$$
1_{|v-w| \leq c l^{-1}} W_{\delta}^{l}(v) \sim 1_{|v-w| \leq c l^{-1}} W_{\delta}^{l}(w) .
$$

Next by the mean value theorem, we see that

$$
\begin{aligned}
& 1_{|v-w| \leq c l^{-1}}\left|\nabla W_{\delta}^{l}(v)-\nabla W_{\delta}^{l}(w)\right| \\
& \leq 1_{|v-w| \leq c l^{-1}} \int_{0}^{1}\left|\left(\nabla^{2} W_{\delta}^{l}\right)(v+t(w-v))\right| d t|v-w| \\
& \leq 1_{|v-w| \leq c l^{-1}} C l^{2} \int_{0}^{1} W_{\delta}^{l}(v+t(w-v))\langle v+t(w-v)\rangle^{-2} d t|v-w|,
\end{aligned}
$$

where we have used the estimate $\left|\nabla_{v}^{2} W_{\delta}^{l}(v)\right| \leq C l^{2} W_{\delta}^{l}(v)\langle v\rangle^{-2}$ which is a consequence of (50) (see below). Notice that $|(v+t(w-v))-v| \leq c l^{-1}$ and $|(v+t(w-v))-w| \leq c l^{-1}$. Then by (49) we obtain that, if $|v-w| \leq c l^{-1}$,

$$
W_{\delta}^{l}(v+t(w-v)) \sim W_{\delta}^{l}(v) \sim W_{\delta}^{l}(w) .
$$

Thus

$$
\begin{aligned}
& 1_{|v-w| \leq c l^{-1}}\left|\nabla W_{\delta}^{l}(v)-\nabla W_{\delta}^{l}(w)\right| \\
& \leq 1_{|v-w| \leq c l^{-1}} C l^{2}|v-w| \min \left\{W_{\delta}^{l}(v), W_{\delta}^{l}(w)\right\} \min \left\{\langle v\rangle^{-2},\langle w\rangle^{-2}\right\},
\end{aligned}
$$

which is enough to prove the claim. 
As a consequence,

$$
\begin{aligned}
\left|I_{\delta}\right| & \leq C l^{2} D(f)^{1 / 2} \eta^{\gamma / 2+2}\left(M_{l}^{\delta}(f)\right)^{1 / 2}\left(\int f(v) W_{\delta}^{l}(v)\langle v\rangle^{-4} d v\right)^{1 / 2} \\
& \leq D(f) M_{l}^{\delta}(f)+C l^{-\gamma}\left(\int f(v) W_{\delta}^{l}(v)\langle v\rangle^{-4} d v\right) .
\end{aligned}
$$

Estimate of $I I_{\delta}$. Inserting the computations

$$
\begin{aligned}
\partial_{j} W_{\delta}^{l}(v)= & l W_{\delta}^{l}(v) \frac{v_{j}}{\langle v\rangle^{2}}-l W_{\delta}^{l}(v) \frac{\delta v_{j}}{1+\delta|v|^{2}} \\
\partial_{i j} W_{\delta}^{l}(v)= & l W_{\delta}^{l}(v) \frac{\delta_{i j}}{\langle v\rangle^{2}}+l(l-2) W_{\delta}^{l}(v) \frac{v_{i} v_{j}}{\langle v\rangle^{4}}-l W_{\delta}^{l}(v) \frac{\delta \delta_{i j}}{1+\delta|v|^{2}} \\
& +l(l+2) W_{\delta}^{l}(v) \frac{\delta^{2} v_{i} v_{j}}{\left(1+\delta|v|^{2}\right)^{2}}-2 l^{2} W_{\delta}^{l}(v) \frac{\delta}{1+\delta|v|^{2}} \frac{v_{i} v_{j}}{\langle v\rangle^{2}},
\end{aligned}
$$

into (48), we have

(51)

$$
\begin{aligned}
I I_{\delta} & =l \iint f(v) f(w) \chi_{\eta}^{c}|v-w|^{\gamma} W_{\delta}^{l}(v)\langle v\rangle^{-2}\left(-2|v|^{2}+2|w|^{2}+(l-2) \frac{|v|^{2}|w|^{2}-(v \cdot w)^{2}}{\langle v\rangle^{2}}\right) d v d w \\
& +l \iint f(v) f(w) \chi_{\eta}^{c}|v-w|^{\gamma} W_{\delta}^{l}(v) \frac{\delta}{1+\delta|v|^{2}}\left(2|v|^{2}-2|w|^{2}+(l+2)\left(|v|^{2}|w|^{2}-(v \cdot w)^{2}\right) \frac{\delta}{1+\delta|v|^{2}}\right) d v d w \\
& -2 l^{2} \iint f(v) f(w) \chi_{\eta}^{c}|v-w|^{\gamma} W_{\delta}^{l}(v) \frac{\delta\langle v\rangle^{-2}}{1+\delta|v|^{2}}\left(|v|^{2}|w|^{2}-(v \cdot w)^{2}\right) d v d w .
\end{aligned}
$$

It is obvious that

$$
\left|I I_{\delta}\right| \leq C l^{2} \iint f(v) f(w) W_{\delta}^{l}(v)\left(1+\langle w\rangle^{2}\right) d v d w .
$$

Then thanks to the estimates of $I_{\delta}$ and $I I_{\delta}$, we get

$$
M_{l}^{\delta}(f(t))-M_{l}^{\delta}\left(f_{0}\right) \leq \int_{0}^{t} D(f(s)) M_{l}^{\delta}(f(s)) d s+C l^{2}\left(M_{2}\left(f_{0}\right)+1\right) \int_{0}^{t} M_{l}^{\delta}(f(s)) d s .
$$

By Gronwall's inequality, we obtain that, for some constant $C>0$ and for all $t \geq 0$,

$$
M_{l}^{\delta}(f(t)) \leq C M_{l}^{\delta}\left(f_{0}\right)+C e^{C l^{2} t},
$$

which first implies that $M_{l}(f)$ is bounded (uniformly locally in time):

$$
M_{l}(f(t)) \leq C M_{l}\left(f_{0}\right)+C e^{C l^{2} t},
$$

thanks to Fatou's Lemma. We shall now use the bound (52) in order to improve the moment estimate. We recall that

$$
\begin{aligned}
M_{l}^{\delta}(f(t))-M_{l}^{\delta}\left(f_{0}\right) & =\int_{0}^{t} I_{\delta}(s) d s+\int_{0}^{t} I I_{\delta}(s) d s \\
& \leq \int_{0}^{t} D(f(s)) M_{l}^{\delta}(f(s)) d s+C l^{-\gamma} \int_{0}^{t} M_{l-4}(f(s)) d s+\int_{0}^{t} I I_{\delta}(s) d s .
\end{aligned}
$$

Then by Gronwall's inequality, we get

$$
M_{l}^{\delta}(f(t)) \leq C M_{l}\left(f_{0}\right)+C l^{-\gamma} \int_{0}^{t} M_{l-4}(f(s)) d s+C \int_{0}^{t} I I_{\delta}(s) d s .
$$


Thanks to (51) and the bound (52), we easily observe that we can apply the dominated convergence theorem for the last term in the right-hand side of eq. (53). We therefore obtain, for all $t \geq 0$,

$$
M_{l}(f(t)) \leq C M_{l}\left(f_{0}\right)+C l^{-\gamma} \int_{0}^{t} M_{l-4}(f(s)) d s+C \int_{0}^{t} I I(s) d s .
$$

From that inequality we can copy the argument used in the first step to give the bound on the term $I I$ and thus obtain the desired estimate. This ends the proof of the lemma.

Corollary 8.1. Consider the same setting as in Lemma 8. Suppose further that $M_{s, \kappa}\left(f_{0}\right)=$ $\int f_{0}(v) e^{\kappa\langle v\rangle^{s}} d v<\infty$ with $\kappa>0$ and $0<s<\frac{\gamma+4}{|\gamma+1|}$, or with $0<\kappa<\frac{1}{e} \frac{|\gamma+1|}{\gamma+4}$ and $s=\frac{\gamma+4}{|\gamma+1|}$. Then there is $C>0$ depending on the parameters $\gamma, s, \kappa$ and the initial mass energy and entropy (that is, depending on $M_{0}(f), M_{2}(f)$ and $H\left(f_{0}\right)$ ) such that

$$
M_{s, \kappa}(f(t)) \leq C M_{s, \kappa}(f(0))+C t, \quad \forall t \geq 0 .
$$

Proof. The proof follows the same arguments as in the proof of Corollary 7.17(1). From Lemma 8 and writing $e^{\kappa\langle v\rangle^{s}}=\sum_{j=0}^{\infty} \kappa^{j} \frac{\langle v\rangle^{j s}}{j !}$ we get

$$
M_{s, \kappa}(f(t))=\sum_{j=0}^{\infty} \frac{\kappa^{j}}{j !} M_{j s}(f(t)) \leq C t \sum_{j=0}^{\infty} \frac{\kappa^{j}}{j !}(s j)^{(s j-6) \frac{|\gamma+1|}{\gamma+4}-\gamma}+C M_{s, \kappa}\left(f_{0}\right) .
$$

We then conclude by observing that

$\beta_{j}:=\frac{\kappa^{j}}{j !}(s j)^{(s j-6) \frac{|\gamma+1|}{\gamma+4}-\gamma}=\left(\kappa s^{s \frac{|\gamma+1|}{\gamma+4}}\right)^{j}(s j)^{-6 \frac{|\gamma+1|}{\gamma+4}-\gamma} \frac{j^{j s \frac{|\gamma+1|}{\gamma+4}}}{j !}, \quad j ! \sim(j / e)^{j} \sqrt{2 \pi j} \quad$ as $\quad j \rightarrow \infty$,

which implies $\sum_{j=0}^{\infty} \beta_{j}<\infty$ under the assumptions of the corollary.

Remark 8.1. If we consider $s+\gamma \geq 0$, the same argument presented in the proof of Corollary 7.1 (2) would give us a uniform in time bound for the moment $M_{s, \alpha}(f(t))$. But the conditions $\gamma \in(-4,-2], 0<s \leq(\gamma+4) /|\gamma+1|$ and $s+\gamma \geq 0$ imply $\gamma=-2$ and $s=2$, so that we recover exactly the result stated in Corollary 7.1. (2) (for $\gamma=-2$ ).

\section{LARGE TIME BEHAVIOUR}

We now turn to the proof of Theorem 2, Before starting it, we state an interpolation lemma.

Lemma 9. (i) Let $r \in] 1,3\left[\right.$, and $\alpha \in \mathbb{R}$. Define $\theta(r, \alpha)=\frac{9(r-1)+2 \alpha}{3-r}$. Then, there exists a constant $C:=C(r)>0$, such that for any $f:=f(v) \geq 0$,

$$
\int_{\mathbb{R}^{3}}\langle v\rangle^{\alpha} f|\log f| d v \leq C\left(M_{\alpha+2}(f)+M_{\theta(r, \alpha)}(f)^{\frac{3-r}{2}}\|f\|_{L_{-3}^{3}\left(\mathbb{R}^{3}\right)}^{\frac{3}{2}(r-1)}+1\right) .
$$

(ii) Let $r \in] 1,3[, s \in] 0,2\left[\right.$ and $\kappa>0$. Then for any $\kappa_{1}>\kappa$ and $\kappa_{2}>2 \kappa /(3-r)$, one can find a constant $C:=C\left(r, \kappa, \kappa_{1}, \kappa_{2}\right)>0$ such that for any $f:=f(v) \geq 0$,

$$
\int_{\mathbb{R}^{3}} e^{\kappa\langle v\rangle^{s}} f|\log f| d v \leq C\left(M_{s, \kappa_{1}}(f)+M_{s, \kappa_{2}}(f)^{\frac{3-r}{2}}\|f\|_{L_{-3}^{3}}^{\frac{3}{2}(r-1)}+1\right) .
$$

Proof. (i) We decompose the integral into

$$
\int\langle v\rangle^{\alpha} f|\log f|=\int\langle v\rangle^{\alpha} f|\log f|\left\{\mathbf{1}_{f>1}+\mathbf{1}_{e^{-|v|^{2}<f \leq 1}}+\mathbf{1}_{0 \leq f \leq e^{-|v|^{2}}}\right\}=: I_{1}+I_{2}+I_{3} .
$$

For the term $I_{1}$, we notice that $f|\log f| \mathbf{1}_{f>1} \leq C(r) f^{r} \mathbf{1}_{f>1}$ (for $\left.r \in\right] 1,3[$, and some constant $C(r)>0)$. 
Thanks to Hölder's inequality, we get

$$
\begin{aligned}
I_{1} & =\int\langle v\rangle^{\alpha} f \log f \mathbf{1}_{f>1} \\
& \leq C(r) \int\langle v\rangle^{\alpha} f^{r}=C(r) \int\langle v\rangle^{\alpha+\frac{9}{2}(r-1)} f^{\frac{1}{2}(3-r)}\langle v\rangle^{-\frac{9}{2}(r-1)} f^{\frac{3}{2}(r-1)} \\
& \leq C(r) M_{\theta(r, \alpha)}(f)^{\frac{3-r}{2}}\|f\|_{L_{-3}^{3}}^{\frac{3}{2}(r-1)} .
\end{aligned}
$$

For $I_{2}$, we use the inequality $|\log f| \mathbf{1}_{e^{-|v|^{2}}<f \leq 1} \leq\langle v\rangle^{2} \mathbf{1}_{e^{-|v|^{2}}<f \leq 1}$, in order to obtain

$$
I_{2} \leq M_{\alpha+2}(f) \text {. }
$$

Finally, for $I_{3}$, we use the estimate $f|\log f| \mathbf{1}_{0 \leq f \leq e^{-|v|^{2}}} \leq C \sqrt{f} \mathbf{1}_{0 \leq f \leq e^{-|v|^{2}}} \leq C e^{-|v|^{2} / 2}$, so that

$$
I_{3} \leq C \int\langle v\rangle^{\alpha} e^{-|v|^{2} / 2} \leq C
$$

for some constant $C>0$.

(ii) We decompose the integral into

$$
\int e^{\kappa\langle v\rangle^{s}} f|\log f|=\int e^{\kappa\langle v\rangle^{s}} f|\log f|\left\{\mathbf{1}_{f>1}+\mathbf{1}_{e^{-|v|^{2}}<f \leq 1}+\mathbf{1}_{0 \leq f \leq e^{-|v|^{2}}}\right\}=: I_{1}^{\prime}+I_{2}^{\prime}+I_{3}^{\prime} .
$$

For the term $I_{1}^{\prime}$, again thanks to Hölder's inequality, we obtain (for any $\left.r \in\right] 1,3[$ and some constant $C(r)>0)$

$$
\begin{aligned}
I_{1}^{\prime} & =\int e^{\kappa\langle v\rangle^{s}} f|\log f| \mathbf{1}_{f>1} \\
& \leq C(r) \int e^{\kappa\langle v\rangle^{s}} f^{r}=C(r) \int\langle v\rangle^{\frac{9}{2}(r-1)} e^{\kappa\langle v\rangle^{s}} f^{(3-r) / 2}\langle v\rangle^{-\frac{9}{2}(r-1)} f^{\frac{3}{2}(r-1)} \\
& \leq C\left(r, \kappa, \kappa_{2}\right) M_{s, \kappa_{2}}(f)^{\frac{3-r}{2}}\|f\|_{L_{-3}^{\frac{3}{2}(r-1)}}^{3} .
\end{aligned}
$$

For $I_{2}^{\prime}$, we get (with constants whose dependence is explicitly stated)

$$
I_{2}^{\prime} \leq C\left(\kappa, \kappa_{1}\right) M_{s, \kappa_{1}}(f) .
$$

Finally, for $I_{3}^{\prime}$, we get

$$
I_{3}^{\prime} \leq C(\kappa) \int e^{\kappa\langle v\rangle^{s}-|v|^{2} / 2} \leq C(\kappa)
$$

From now on we consider a global (equivalently $H$ - or weak) solution $f:=f(t, v) \geq 0$ to the spatially homogeneous Landau equation with Coulomb potential (11), associated to nonnegative initial data $f_{0} \in L_{2}^{1} \cap L \log L\left(\mathbb{R}^{3}\right)$ satisfying the normalization (12).

We shall use in the sequel the following properties of such a solution:

- the conservation of mass, momentum and energy (10), more precisely

$$
\int f(t, v) d v=1, \quad \int f(t, v) v d v=0, \quad \int f(t, v)|v|^{2} d v=3, \quad \forall t \geq 0
$$

- the moments estimates of Lemma 8 and Corollary 8.1

- the entropy-entropy dissipation inequality

$$
H(f(t))+\int_{0}^{t} D(f(\tau)) d \tau \leq H\left(f_{0}\right) \leq C_{0}, \quad \forall t \geq 0
$$

- $f$ satisfies estimate (13).

We now start the: 
Proof of Theorem 固(i). Recall that we assume that $f_{0} \in L_{\ell}^{1}\left(\mathbb{R}^{3}\right)$ with $\ell>\frac{19}{2}$, which can be rewritten as $\ell=\frac{9}{2}+\frac{3 k}{2}$ and $k>\frac{10}{3}$.

We split the proof into four steps. All constants $C>0$ in the proof of this part of the theorem are simply written by $C$ (and can change from line to line), but in fact depend on $\ell, M_{\ell}\left(f_{0}\right), \bar{H}$, where $\int f_{0} \log f_{0} d v \leq \bar{H}$ (if $f_{0}$ were not normalized, they also would depend on the mass and energy of $f_{0}$ ).

Step 1. First of all, we obtain from Lemma 8 (with $\gamma=-3$ ) that $M_{\ell}(f(t)) \leq C(1+t)$, and interpolating this result with the conservation of the energy $M_{2}(f(t))=M_{2}\left(f_{0}\right)=4$ for any $t \geq 0$, it follows

$$
M_{5}(f(t)) \leq C(1+t)^{\frac{3}{\ell-2}}
$$

since $\ell>5$.

Step 2. As a consequence we can write, using the entropy dissipation inequality (21) of Corollary 1.1 with some $R(t)>0$ to be chosen later (note that $C$ depends on $k$ below),

$$
\begin{aligned}
D(f(t)) \geq & C(1+t)^{-\frac{3}{\ell-2}} R(t)^{-3} H(f(t) \mid \mu) \\
& -C(1+t)^{-\frac{3}{\ell-2}} R(t)^{-3-k}\left\{\int_{\mathbb{R}^{3}} f(t)|\log f(t)|\langle v\rangle^{k} d v+M_{k+2}(f(t))+1\right\} .
\end{aligned}
$$

Thanks to Lemma 9f(i) with $\alpha=k, r=5 / 3$, so that $\frac{3}{2}(r-1)=1$ and $\theta(r, k)=\frac{9+3 k}{2}$, we get

$$
\begin{aligned}
D(f(t)) \geq & C(1+t)^{-\frac{3}{\ell-2}} R(t)^{-3} H(f(t) \mid \mu) \\
& -C(1+t)^{-\frac{3}{\ell-2}} R(t)^{-3-k}\left(M_{k+2}(f(t))+M_{\frac{9+3 k}{2}}(f(t))^{\frac{2}{3}}\|f(t)\|_{L_{-3}^{3}}+1\right) .
\end{aligned}
$$

Thanks to (13), we know that

$$
M_{\frac{9+3 k}{2}}(f(t))^{\frac{2}{3}}\|f(t)\|_{L_{-3}^{3}} \leq C M_{\frac{9+3 k}{2}}(f(t))^{\frac{2}{3}}(D(f(t))+1),
$$

thus it follows

$$
\begin{aligned}
(1+C & \left.(1+t)^{-\frac{3}{\ell-2}} M_{\frac{9+3 k}{2}}(f(t))^{\frac{2}{3}} R(t)^{-3-k}\right) D(f(t)) \\
\geq & C(1+t)^{-\frac{3}{\ell-2}} R(t)^{-3} H(f(t) \mid \mu) \\
& -C(1+t)^{-\frac{3}{\ell-2}} R(t)^{-3-k} M_{k+2}(f(t)) \\
& -C(1+t)^{-\frac{3}{\ell-2}} R(t)^{-3-k} M_{\frac{9+3 k}{2}}(f(t))^{\frac{2}{3}} .
\end{aligned}
$$

Step 3. We now choose $R(t)=(1+t)^{\nu}$ for some $\left.\nu \in\right] 0, \frac{1}{3}\left(\frac{(3 k-1)}{(3 k+5)}\left[\right.\right.$ such that $\nu k>\frac{2}{3}$. Note that this is possible whenever $\frac{1}{3} \frac{(3 k-1)}{(3 k+5)} k>\frac{2}{3}$, which is implied by the condition $k>\frac{10}{3}$.

Using Lemma 8 (with $\gamma=-3$ ) again and interpolating the estimate with the conservation of the energy as in step 1 , we have ( since $\frac{9+3 k}{2}=\ell$ )

$$
M_{k+2}(f(t)) \leq C(1+t)^{\frac{k}{\ell-2}}, \quad\left[M_{\frac{9+3 k}{2}}(f(t))\right]^{\frac{2}{3}} \leq C(1+t)^{\frac{2}{3}} .
$$

Therefore, noticing that

$$
C(1+t)^{-\frac{3}{\ell-2}} M_{\frac{9+3 k}{2}}(f)^{\frac{2}{3}} R(t)^{-3-k} \leq C,
$$


we end up with

$$
\begin{aligned}
D(f(t)) & \geq \frac{C H(f(t) \mid \mu)}{(1+t)^{3 \nu+\frac{6}{5+3 k}}}-\frac{C}{(1+t)^{3 \nu+k \nu+\frac{6}{5+3 k}-\frac{2 k}{5+3 k}}}-\frac{C}{(1+t)^{3 \nu+k \nu+\frac{6}{5+3 k}-\frac{2}{3}}} \\
& \geq \frac{C H(f(t) \mid \mu)}{(1+t)^{3 \nu+\frac{6}{5+3 k}}}-\frac{C}{(1+t)^{3 \nu+k \nu+\frac{6}{5+3 k}-\frac{2}{3}}} .
\end{aligned}
$$

Step 4. Denoting $x(t):=H(f(t) \mid \mu), a:=3 \nu+\frac{6}{5+3 k}$ and $b:=3 \nu+\nu k+\frac{6}{5+3 k}-\frac{2}{3}$, we plug the last estimate into (54) and obtain the following inequality (we denote by $C_{1}>0$ and $C_{2}>0$ two different constants in order to avoid confusions)

$$
x(t)+C_{1} \int_{0}^{t} \frac{x(\tau)}{(1+\tau)^{a}} d \tau \leq C_{0}+C_{2} \int_{0}^{t}(1+\tau)^{-b} d \tau,
$$

with $0<a<1$ (because $\nu<\frac{1}{3} \frac{3 k-1}{3 k+5}$ ) and $b>a$ (because $\frac{1}{3} \frac{(3 k-1)}{(3 k+5)} k>\frac{2}{3}$, since $k>\frac{10}{3}$ ).

We recall that the generalized Gronwall inequality (see e.g. [34])

$$
u(t) \leq \phi(t)+\int_{0}^{t} \lambda(\tau) u(\tau) d \tau
$$

implies

$$
u(t) \leq \phi(0) e^{\int_{0}^{t} \lambda(\tau) d \tau}+\int_{0}^{t} e^{\int_{\tau}^{t} \lambda(\sigma) d \sigma} \frac{d \phi(\tau)}{d \tau} d \tau
$$

that we apply to (55) and obtain

$$
x(t) \leq C_{0} e^{-C_{1} \frac{(1+t)^{1-a}}{1-a}}+C_{2} e^{-C_{1} \frac{(1+t)^{1-a}}{1-a}} \int_{0}^{t}(1+\tau)^{-b} e^{C_{1} \frac{(1+\tau)^{1-a}}{1-a}} d \tau .
$$

Then we observe that thanks to an integration by parts,

$$
\begin{gathered}
e^{-C_{1} \frac{(1+t)^{1-a}}{1-a}} \int_{0}^{t}(1+\tau)^{-b} e^{C_{1} \frac{(1+\tau)^{1-a}}{1-a}} d \tau \\
=e^{-C_{1} \frac{(1+t)^{1-a}}{1-a}}\left[C_{1}^{-1} e^{C_{1} \frac{(1+t)^{1-a}}{1-a}}(1+t)^{a-b}-C_{1}^{-1} e^{\frac{1}{1-a}}+C_{1}^{-1}(b-a) \int_{0}^{t}(1+\sigma)^{a-b-1} e^{C_{1} \frac{(1+\sigma)^{1-a}}{1-a}} d \sigma\right] \\
\leq C_{1}^{-1}(1+t)^{a-b}+C_{1}^{-1}(b-a) e^{-C_{1} \frac{(1+t)^{1-a}}{1-a}} \int_{0}^{t}(1+\sigma)^{a-b-1} e^{C_{1} \frac{(1+\sigma)^{1-a}}{1-a}} d \sigma \\
\leq C_{1}^{-1}(1+t)^{a-b}+C_{1}^{-1}(b-a) \frac{t}{2} e^{-C_{1} \frac{(1+t)^{1-a}}{1-a}}(1+t / 2)^{a-b-1} e^{C_{1} \frac{(1+t / 2)^{1-a}}{1-a}}+C_{1}^{-1}(b-a) \frac{t}{2}(1+t / 2)^{a-b-1} .
\end{gathered}
$$

We therefore see that

$$
x(t) \leq C(1+t)^{-(b-a)}=C(1+t)^{-\left(\nu k-\frac{2}{3}\right)} .
$$

This entails that $H(f(t) \mid \mu) \leq C(1+t)^{-\beta}$ for all $\left.\beta \in\right] 0, \frac{k}{3} \frac{(3 k-1)}{(3 k+5)}-\frac{2}{3}[$, and this ends the proof of Theorem 2r(i).

We now turn to the

Proof of Theorem 国(ii). In the sequel, the constant denoted by $C$ in fact depends on $\kappa, s$, and $\bar{H}$. We split the proof into the same four steps as in the proof of Theorem 2.(i).

Step 1. From Corollary 8.1 we get the estimate

$$
M_{s, \kappa}(f(t)) \leq C(1+t) .
$$

Interpolating this estimate with the conservation of the energy $M_{2}(f(t))=M_{2}\left(f_{0}\right)=4$ (for all $t \geq 0$ ), we claim that

$$
M_{5}(f(t)) \leq C \log ^{3 / s}(1+t)
$$


Indeed we can write, for any $r>(6 /(\kappa s))^{1 / s}$,

$$
\begin{aligned}
\int\langle v\rangle^{5} f(t) & =\int_{\langle v\rangle \leq r}\langle v\rangle^{5} f(t)+\int_{\langle v\rangle>r}\langle v\rangle^{5} f(t) \\
& \leq r^{3} M_{2}(f(t))+r^{3} e^{-\frac{\kappa}{2} r^{s}} \int_{\langle v\rangle>r}\langle v\rangle^{2} e^{\frac{\kappa}{2}\langle v\rangle^{s}} f(t) \\
& \leq C r^{3}\left(1+e^{-\frac{\kappa}{2} r^{s}} M_{s, \kappa}(f(t))\right) .
\end{aligned}
$$

We choose then $r=\sup \left[\left(\frac{2}{\kappa} \log M_{s, \kappa}(f(t))\right)^{1 / s},(6 /(\kappa s))^{1 / s}\right]$, which concludes the claim.

Step 2. Using the entropy dissipation inequality (21) of Corollary 1.1 we can argue as in the proof of Theorem 2 (i) above and obtain, for any $\left.\kappa_{0} \in\right] 0, \kappa[$ and some $R(t)>0$ to be chosen later:

$$
\begin{aligned}
D(f(t)) \geq & C \log ^{-3 / s}(1+t) R(t)^{-3} H(f(t) \mid \mu) \\
& -C \log ^{-3 / s}(1+t) R(t)^{-3} e^{-\kappa_{0} R(t)^{s}}\left\{\int e^{\kappa_{0}\langle v\rangle^{s}} f(t)|\log f(t)|+\int\langle v\rangle^{2} e^{\kappa_{0}\langle v\rangle^{s}} f(t)+1\right\} .
\end{aligned}
$$

Then, for $r \in] 1,3\left[, \kappa_{1}>\kappa_{0}, \kappa_{2}>2 \kappa_{0} /(3-r)\right.$, it follows from Lemma 9 and the bound (13) that

$$
\begin{aligned}
D(f(t)) \geq & C \log ^{-3 / s}(1+t) R(t)^{-3} H(f(t) \mid \mu) \\
& -C \log ^{-3 / s}(1+t) R(t)^{-3} e^{-\kappa_{0} R(t)^{s}}\left[2 M_{s, \kappa_{1}}(f(t))+1+M_{s, \kappa_{2}}(f(t))^{(3-r) / 2}\|f(t)\|_{L_{-3}^{3}}^{\frac{3}{2}(r-1)}\right] .
\end{aligned}
$$

As a consequence, considering $r=5 / 3$, and $\left.\kappa_{1} \in\right] \kappa_{0}, \kappa\left[, \kappa_{2}>\frac{3}{2} \kappa_{0}\right.$, we get

$$
\begin{gathered}
D(f(t))\left(1+C \log ^{-3 / s}(1+t) R(t)^{-3} e^{-\kappa_{0} R(t)^{s}} M_{s, \kappa_{2}}(f(t))^{2 / 3}\right) \\
\geq \frac{C H(f(t) \mid \mu)}{R(t)^{3} \log ^{3 / s}(1+t)}-\frac{C(1+t)}{e^{\kappa_{0} R(t)^{s}} R(t)^{3} \log ^{3 / s}(1+t)} .
\end{gathered}
$$

Step 3. We now choose $R(t)=(1+t)^{\frac{1}{3+s}}(\log (1+t))^{-\frac{3+q s}{3 s}}$, for some $q \in \mathbb{R}$ to be chosen later, so that, taking $\left.\kappa_{0} \in\right] 0, \frac{2}{3} \kappa\left[\right.$ and $\left.\kappa_{2} \in\right] \frac{3}{2} \kappa_{0}, \kappa[$,

$$
C \log ^{-3 / s}(1+t) R(t)^{-3} e^{-\kappa_{0} R(t)^{s}} M_{s, \kappa_{2}}(f(t))^{2 / 3} \leq C .
$$

Denoting $x(t):=H(f(t) \mid \mu)$ and gathering the previous estimates together with (54), we see that (denoting by $C_{1}, C_{2}>0$ the constants to avoid confusions)

$$
\begin{aligned}
x(t) & +C_{1} \int_{0}^{t} \frac{x(\tau)}{(1+\tau)^{\frac{3}{3+s}}(\log (1+\tau))^{-q}} d \tau \\
& \leq C_{0}+C_{2} \int_{0}^{t} e^{-\kappa_{0}(1+\tau)^{\frac{s}{3+s}}(\log (1+\tau))^{-\frac{3+q s}{3}}}(1+\tau)^{\frac{s}{3+s}}(\log (1+\tau))^{q} d \tau,
\end{aligned}
$$

thus by the generalized Gronwall's inequality it follows, denoting $A(t):=\int_{0}^{t}(1+\tau)^{-\frac{3}{3+s}}(\log (1+$ $\tau))^{q} d \tau$,

$x(t) \leq C_{0} e^{-C_{1} A(t)}+C_{2} e^{-C_{1} A(t)} \int_{0}^{t} e^{C_{1} A(\tau)}(1+\tau)^{\frac{s}{3+s}}(\log (1+\tau))^{q} e^{-\kappa_{0}(1+\tau)^{\frac{s}{3+s}}(\log (1+\tau))^{-\frac{3+q s}{3}}} d \tau$.

We can now complete the proof by some elementary computations. We observe that (thanks to an integration by parts) for all $a>-1$ and $b \in \mathbb{R}$,

$$
\int_{0}^{t}(1+\sigma)^{a}(\log (1+\sigma))^{b} d \sigma=\frac{(1+t)^{a+1}}{a+1}(\log (1+t))^{b}+O\left((1+t)^{a+1}(\log (1+t))^{b-1}\right),
$$


so that (for $t$ large enough)

$$
2(1+t)^{\frac{s}{3+s}}(\log (1+t))^{q} \geq \int_{0}^{t}(1+\sigma)^{-\frac{3}{3+s}}(\log (1+\sigma))^{q} d \sigma \geq(1+t)^{\frac{s}{3+s}}(\log (1+t))^{q} .
$$

We hence split

$$
\begin{aligned}
x(t) \leq & C_{0} e^{-C_{1} A(t)} \\
& +C_{2} e^{-C_{1} A(t)} \int_{0}^{t / n} e^{C_{1} A(\tau)}(1+\tau)^{\frac{s}{3+s}}(\log (1+\tau))^{q} e^{-\kappa_{0}(1+\tau)^{\frac{s}{3+s}}(\log (1+\tau))^{-\frac{3+q s}{3}}} d \tau \\
& +C_{2} e^{-C_{1} A(t)} \int_{t / n}^{t} e^{C_{1} A(\tau)}(1+\tau)^{\frac{s}{3+s}}(\log (1+\tau))^{q} e^{-\kappa_{0}(1+\tau)^{\frac{s}{3+s}}(\log (1+\tau))^{-\frac{3+q s}{3}}} d \tau \\
= & : e^{-C_{1} A(t)} x(0)+I_{1}+I_{2},
\end{aligned}
$$

for some $n>0$ to be chosen large enough. Thanks to (56), we easily get

$$
C_{0} e^{-C_{1} A(t)} \leq C_{0} e^{-C_{1}(1+t)^{\frac{s}{3+s}}(\log (1+t))^{q}} .
$$

Moreover we write for the term $I_{1}$, using (56),

$$
\begin{aligned}
I_{1} & \leq C_{2} e^{-C_{1}(A(t)-A(t / n))} \int_{0}^{t / n}(1+\tau)^{\frac{s}{3+s}}(\log (1+\tau))^{q} e^{-\kappa_{0}(1+\tau)^{\frac{s}{3+s}}(\log (1+\tau))^{-\frac{3+q s}{3}}} d \tau \\
& \leq C e^{-C_{1}(A(t)-A(t / n))} \leq C e^{-C_{1}(1+t)^{\frac{s}{3+s}}(\log (1+t))^{q}+2 C_{1}(1+t / n)^{\frac{3}{3+s}}(\log (1+t / n))^{q}} \\
& \leq C e^{-C_{1}(1+t)^{\frac{s}{3+s}}(\log (1+t))^{q}\left(1-2 n^{-\frac{3}{(3+s)}}\right)} \leq C e^{-\frac{C_{1}}{2}(1+t)^{\frac{s}{3+s}}(\log (1+t))^{q}},
\end{aligned}
$$

when $n>0$ is chosen large enough (i.e. such that $1-\frac{2}{n^{3 /(3+s)}} \geq 1 / 2$ ). For the other term, we have

$$
\begin{aligned}
I_{2} & \leq C_{2} \int_{t / n}^{t}(1+\tau)^{\frac{s}{3+s}}(\log (1+\tau))^{q} e^{-\kappa_{0}(1+\tau)^{\frac{s}{3+s}}(\log (1+\tau))^{-\frac{3+q s}{3}}} d \tau \\
& \leq C e^{-\frac{\kappa_{0}}{2}(1+t / n)^{\frac{s}{3+s}}(\log (1+t / n))^{-\frac{3+q s}{3}}} \int_{t / n}^{t}(1+\tau)^{\frac{s}{3+s}}(\log (1+\tau))^{q} e^{-\frac{\kappa_{0}}{2}(1+\tau)^{\frac{s}{3+s}}(\log (1+\tau))^{-\frac{3+q s}{3}}} d \tau \\
& \leq C e^{-c \frac{\kappa_{0}}{2}(1+t)^{\frac{s}{3+s}}(\log (1+t))^{-\frac{3+q s}{3}}},
\end{aligned}
$$

for some constants $c, C>0$.

Finally, taking $-\frac{3+q s}{3}=q$, that is $q=-\frac{3}{3+s}$, we deduce that there are constants $C, c>0$ such that

which completes the proof.

$$
x(t) \leq C e^{-c(1+t)^{\frac{s}{3+s}}(\log (1+t))^{-\frac{3}{3+s}}},
$$

Acknowledgement: The research leading to this paper was funded by the French "ANR blanche" project Kibord: ANR-13-BS01-0004. K.C. is supported by the Fondation Mathématique Jacques Hadamard.

\section{REFERENCES}

[1] M. Aizenman and T. Bak, Convergence to equilibrium in a system of reacting polymers, Comm. Math. Phys., 65:203-230, 1979.

[2] R. Alexandre, J. Liao, and C. Lin. Some a priori estimates for the homogeneous Landau equation with soft potentials. arXiv:1302.1814

[3] R. Alexandre, Y. Morimoto, S. Ukai, C.-J. Xu and T. Yang. The Boltzmann equation without angular cutoff in the whole space: I, Global existence for soft potential. J. Funct. Anal., 262(3):915-1010, 2012.

[4] R. Alexandre, Y. Morimoto, S. Ukai, C.-J. Xu and T. Yang. The Boltzmann equation without angular cutoff in the whole space: II, Global existence for hard potential. Anal. Appl. (Singap.), 9(2):113-134, 2011. 
[5] A. Arnold, P. Markowich, G. Toscani and A. Unterreiter. On convex Sobolev inequalities and the rate of convergence to equilibrium for Fokker-Planck type equations. Comm. PDE, 26(1-2):43-100, 2001.

[6] A.A. Arsenev and N.V. Peskov. On the existence of a generalized solution of Landau's equation. Z. Vycisl. Mat. i Mat. Fiz., 17:1063-1068, 1977.

[7] D. Bakry and M. Emery. Hypercontractivité de semi-groupes de diffusion. C.R. Acad. Sc. Paris, t. 299, Série I 15, 1984.

[8] K. Carrapatoso. Exponential convergence to equilibrium for the homogeneous Landau equation with hard potentials. Bull. Sci. Math., 139(7):777-805, 2015.

[9] K. Carrapatoso. On the rate of convergence to equilibrium for the homogeneous Landau equation with soft potentials. J. Math. Pures Appl., 104(2):276-310, 2015.

[10] K. Carrapatoso, I. Tristani and K.-C. Wu. Cauchy problem and exponential stability for the inhomogeneous Landau equation. Preprint arXiv:1504.04124

[11] C. Cercignani. $H$-theorem and trend to equilibrium in the kinetic theory of gases, Arch. Mech. (Arch. Mech. Stos.), 34:231-241 (1983), 1982.

[12] S. Chapman, T.G. Cowling. The mathematical theory of non-uniform gases, Cambridge Univ. Press., London, (1952).

[13] I. Csiszar. Information-type measures of difference of probability distributions and indirect observations, Stud. Sci. Math. Hung., 2:299-318, 1967.

[14] L. Desvillettes. Entropy dissipation estimates for the Landau equation in the Coulomb case and applications. J. Functional Anal., 269(5):1359-1403, 2015.

[15] L. Desvillettes. Entropy dissipation rate and convergence in kinetic equations. Comm. Math. Phys., 123(4):687-702, 1989.

[16] L. Desvillettes. Entropy dissipation estimates for the Landau equation: General cross sections. Accepted for publication in the Proceedings of the workshop PSPDE III, Braga, Dec. 2014.

[17] L. Desvillettes and K. Fellner. Exponential Decay toward Equilibrium via Entropy Methods for ReactionDiffusion Equations, J. Math. Anal. Appl., 319:157-176, 2006.

[18] L. Desvillettes and K. Fellner. Entropy methods for reaction-diffusion equations: slowly growing a priori bounds. Revista Matemática Iberoamericana, 24(2):407-431, 2008.

[19] L. Desvillettes, C. Mouhot and C. Villani. Celebrating Cercignani's conjecture for the Boltzmann equation. Kinet. Relat. Models, 4:277-294, 2011.

[20] L. Desvillettes and C. Villani. On the spatially homogeneous Landau equation for hard potentials. Part I. Existence, uniqueness and smoothness. Commun. Partial Differential Equations, 25(1-2):179-259, 2000.

[21] L. Desvillettes and C. Villani. On the spatially homogeneous Landau equation for hard potentials. Part II. H-Theorem and applications. Commun. Partial Differential Equations, 25(1-2):261-298, 2000.

[22] L. Desvillettes and C. Villani. On the trend to global equilibrium for spatially inhomogeneous kinetic systems: the Boltzmann equation. Invent. Math., 159(2):245-316, 2005.

[23] N. Fournier. Uniqueness of bounded solutions for the homogeneous Landau equation with a Coulomb potential. Commun. Math. Phys., 299:765-782, 2010.

[24] P.T. Gressman and R.M. Strain Global classical solutions of the Boltzmann equation without angular cut-off. J. Amer. Math. Soc., 24:771-847, 2011.

[25] Y. Guo. The Landau equation in a periodic box. Comm. Math. Phys., 231:391-434, 2002.

[26] L. He and X. Yang. Well-posedness and asymptotics of grazing collisions limit of Boltzmann equation with Coulomb interaction. SIAM J. Math. Anal., 46(6):4104-4165, 2014.

[27] S. Kullback. A lower bound for discrimination information in terms of variation, IEEE Trans. Inf. The., 4:126-127, 1967.

[28] E.M. Lifschitz and L.P. Pitaevskii. Physical kinetics. Perg. Press., Oxford, 1981.

[29] C. Mouhot and L. Neumann. Quantitative perturbative study of convergence to equilibrium for collisional kinetic models in the torus. Nonlinearity, 19(4):969-998, 2006.

[30] R.M. Strain and Y. Guo. Almost exponential decay near Maxwellian. Comm. Partial Differential Equations, 31(1-3):417-429, 2006

[31] R.M. Strain and Y. Guo. Exponential decay for soft potentials near Maxwellian. Arch. Ration. Mech. Anal., 187(2):287-339, 2008.

[32] G. Toscani. Entropy production and the rate of convergence to equilibrium for the Fokker-Planck equation, Quart. Appl. Math., 57:521-541, 1999.

[33] G. Toscani and C. Villani. On the trend to equilibrium for some dissipative systems with slowly increasing a priori bounds. J. Statist. Phys., 98(5/6):1279-1309, 2000.

[34] G. Toscani and C. Villani. Probability metrics and uniqueness of the solution to the Boltzmann equation for a Maxwell gas, J. Statist. Phys., 94(3-4):619-637, 1999.

[35] G. Toscani and C. Villani. Sharp entropy dissipation bounds and explicit rate of trend to equilibrium for the spatially homogeneous Boltzmann equation, Comm. Math. Phys., 203:667-706, 1999. 
[36] C. Villani. On a new class of weak solutions to the spatially homogeneous Boltzmann and Landau equations. Arch. Rat. Mech. Anal., 143(3):273-307, 1998.

[37] C. Villani. On the Landau equation: weak stability, global existence. Adv. Diff. Eq., 1(5):793-816, 1996.

[38] C. Villani. A review of mathematical topics in collisional kinetic theory. In Handbook of mathematical fluid dynamics, Vol. I, pages 71-305. North-Holland, Amsterdam, 2002.

[39] C. Villani. Contributions à l'étude mathématique des équations de Boltzmann et de Landau en théorie cinétique des gaz et des plasmas. PhD thesis, Université Paris-Dauphine, 1998.

[40] K.-C. Wu. Global in time estimates for the spatially homogeneous Landau equation with soft potentials. $J$. Funct. Anal., 266:3134-3155, 2014.

[41] H. Yu. The exponential decay of global solutions to the generalized Landau equation near Maxwellians. Quart. Appl. Math., 64(1):29-39, 2006.

(Kleber Carrapatoso) Centre de Mathématiques et de Leurs Applications, EnS Cachan, CNRS, Université Paris-Saclay, F-94235 Cachan, France.

E-mail address: carrapatoso@cmla.ens-cachan.fr

(Laurent Desvillettes) Univ. Paris Diderot, Sorbonne Paris Cité, Institut de Mathématiques de Jussieu - Paris Rive Gauche, UMr 7586, CNRS, Sorbonne Universités, UPMC Univ. Paris 06, F-75013, Paris, France.

E-mail address: desvillettes@math.univ-paris-diderot.fr

(Lingbing He) Department of Mathematical Sciences, Tsinghua University, Beijng, 100084, P.R. China.

E-mail address: lbhe@math.tsinghua.edu.cn 